\title{
Droplet velocity and diameter distributions in flash boiling liquid nitrogen jets by means of phase Doppler diagnostics
}

\author{
Andreas Rees $^{1}\left(\mathbb{0} \cdot\right.$ Lucio Araneo $^{2} \cdot$ Heiko Salzmann $^{1} \cdot$ Grazia Lamanna $^{3} \cdot$ Joachim Sender $^{1} \cdot$ Michael Oschwald $^{1,4}$
}

Received: 27 April 2020 / Revised: 26 June 2020 / Accepted: 22 July 2020 / Published online: 3 August 2020

(c) The Author(s) 2020

\begin{abstract}
Due to current and future environmental and safety issues in space propulsion, typical propellants for upper stage or satellite rocket engines such as the toxic hydrazine are going to be replaced by green propellants like the combination of liquid oxygen and hydrogen or methane. The injection of that kind of cryogenic fluids into the vacuum atmosphere of space leads to a superheated state, which results in a sudden and eruptive atomization due to flash boiling. For a detailed experimental investigation of superheated cryogenic fluids, the new cryogenic test bench M3.3 with a temperature controlled injection system was built at DLR Lampoldshausen. After a first test campaign with high-speed shadowgraphy of flash boiling liquid nitrogen sprays, a laser-based Phase Doppler system was set-up to determine the spatial distributions of droplet velocities and diameters in highly superheated sprays. The spatial distributions revealed a core region with high mean velocities close to the injector orifice. With increasing distance from the injector orifice, the sprays develop a more and more monodisperse pattern. These distributions also showed that atomization due to flash boiling generates finer sprays with growing degrees of superheat. In certain spray regions, two droplet populations varying in their direction of motion, velocity and diameter due to possible recirculation zones were observed. The experimental data of flash boiling liquid nitrogen generated within this study provide a comprehensive data base for the validation of numerical models and further numerical investigations.
\end{abstract}

\section{Graphic abstract}
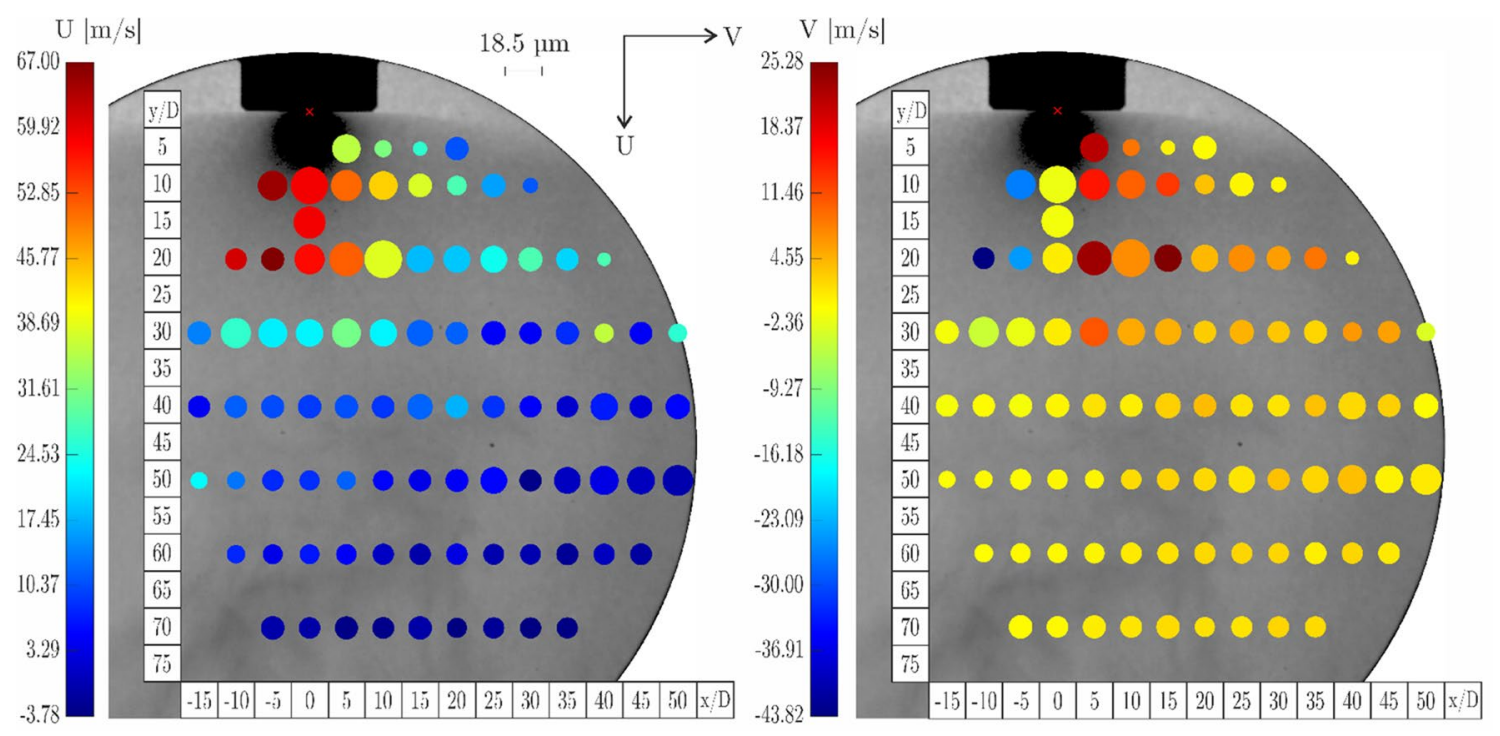

Andreas Rees

andreas.rees@dlr.de

Extended author information available on the last page of the article 


\section{Introduction}

Technology development for propulsion systems of upper stages like the cryogenic Ariane 6 upper stage engine Vinci or for future cryogenic thrusters in reaction control or orbital and manoeuvring systems is driven by the invention of new, green propellants to substitute hydrazine, and by new ignition technologies like laser ignition (Manfletti 2014). At high-altitude conditions prior to ignition the liquid propellants are injected into the combustor at near-vacuum conditions. This means that the ambient pressure $p_{\infty}$ is lower than the liquid's saturation pressure $p_{\text {sat }}\left(T_{\text {inj }}\right)$ at the injection temperature $T_{\text {inj. }}$. The sudden pressure drop at injection leads to a superheated liquid in a metastable thermodynamic state. The injection of highly superheated liquids results in a fast expansion and eruptive evaporation, a process called flash boiling or flash evaporation. The evaporating gases raise the pressure inside the combustion chamber until the equilibrium pressure shortly before ignition is reached. To know the composition of the propellants in the combustion chamber related to phase, species and temperature distribution is important for determining the parameters for a successful ignition and for avoiding destructive pressure peaks.

\subsection{Flash boiling}

The dominating parameters for the flash boiling phenomenon in a given liquid are the injection temperature $T_{\text {inj }}$ and the back pressure $p_{\mathrm{ch}}$, which can be a near-vacuum chamber pressure or atmospheric conditions. According to Fig. 1, they both define the degree of superheat of the injected liquid in terms of the pressure ratio

$R_{\mathrm{p}}=\frac{p_{\mathrm{sat}}\left(T_{\mathrm{inj}}\right)}{p_{\mathrm{ch}}}$

or the ratio of temperature differences

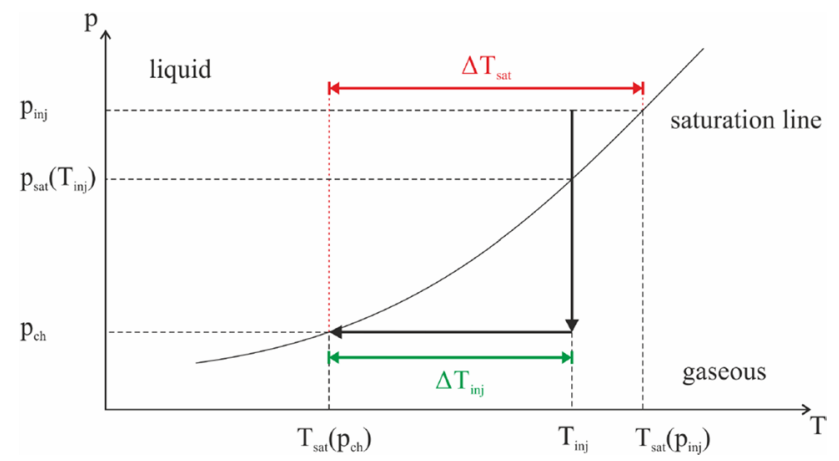

Fig. 1 Phase diagram of a superheated liquid with adiabatic depressurization
$\Delta T^{*}=\frac{\Delta T_{\text {inj }}}{\Delta T_{\text {sat }}}=\frac{T_{\text {inj }}-T_{\text {sat }}\left(p_{\text {ch }}\right)}{T_{\text {sat }}\left(p_{\text {inj }}\right)-T_{\text {sat }}\left(p_{\mathrm{ch}}\right)}$

with the saturation pressure $p_{\text {sat }}\left(T_{\text {inj }}\right)$ at the injection temperature $T_{\text {inj }}$, the saturation temperature $T_{\text {sat }}\left(p_{\mathrm{ch}}\right)$ at the chamber pressure $p_{\text {ch }}$ and the saturation temperature $T_{\text {sat }}\left(p_{\text {inj }}\right)$ at the injection pressure $p_{\text {inj. }}$. A superheated liquid jet with a high degree of superheat is atomized close to or already in the injector nozzle due to vaporization and produces a fine spray with a big opening angle and small droplets. The influence of aerodynamical processes on the primary atomization can be neglected in this kind of jets (Zeng and Lee 2001). The vaporization and expansion of a flash boiling spray leads to a cool-down to reach a new equilibrium state at the saturation temperature $T_{\mathrm{sat}}\left(p_{\mathrm{ch}}\right)$. These non-equilibrium processes are quite complex and need experimental data for further modeling.

\subsection{State of research}

Flash boiling is observed and investigated since the beginning of the twentieth century (Meyer 1911; Wismer 1921). In the last 3 decades, there have been increased efforts of the automotive industry to investigate flash boiling processes of storable liquids typical for the injection into gasoline or diesel engines (Senda et al. 1994; Gemci et al. 2004; Senda et al. 2008; Aleiferis et al. 2010; Araneo and Dondè 2017, 2019). Pre-heating the fuel to reach the superheated condition causes flash boiling during the injection which leads to a finer and wider atomization in the combustion chamber. This increases the efficiency and reduces exhaust emissions (Senda et al. 2008). Compared with cavitation in the injector nozzle flash boiling was found to be the dominant process for fuel atomization (Aleiferis et al. 2010). Adding small amounts of low boiling liquids to the actual fuel can be used to improve atomization by flash boiling (Gemci et al. 2004). Araneo and Dondè (2017) investigated various hydrocarbon sprays injected with a multi-hole injector. By means of backlight shadowgraphy, a relationship between the spray angle and the injection conditions were found. The same authors did Phase Doppler Anemometry (PDA) measurements in hydrocarbon sprays with dissolved air and the resulting sprays showed similar features like flash boiling sprays (Araneo and Dondè 2019). First models were developed for flash boiling hydrocarbon sprays to predict the nucleation rates and resulting droplet sizes (Senda et al. 1994) and for water sprays to predict the liquid superheat with the help of the depressurization transient (Elias and Chambré 1993). Further studies about flash boiling processes can be found concerning the safety field in process technology or chemical and nuclear industry, where storable fluids such as hydrocarbons, water, ethanol or refrigerants like R-134A were used 
(Yildiz et al. 2002; Reitz 1990; Simões-Moreira et al. 2002; Elias and Chambré 1993; Cleary et al. 2007). It was found that not only the degree of superheat determines the intensity of flash boiling but also injection conditions like the injection pressure or the injector diameter (Yildiz et al. 2002). By the means of shadowgraphy, a remaining liquid core in flash boiling sprays was visualized, where the jet break-up due to phase change takes place in a radial direction (Reitz 1990; Simões-Moreira et al. 2002). Transition correlations depending on the dimensionless Weber and Jakob numbers were empirically developed for superheated water leaking into the atmosphere to subdivide the resulting sprays into an aerodynamical break-up region, a transition region and a fully flashing region (Cleary et al. 2007; Witlox et al. 2007). The validity range of these correlations was successfully expanded for the fluids iso-octane, acetone and ethanol by Lamanna et al. (2014). In the same study, an onset criterion, which links flash boiling with the classical nucleation theory, for the flashing regimes was developed, and a model for predicting the spray angle in the near-nozzle region by the degree of superheat and the dimensionless surface tension was generated. In contrast to storable fluids, flash boiling of cryogenic liquids is much less investigated due to significantly harder experimental conditions. Within an experimental study at DLR Lampoldshausen about laser ignition in a model rocket combustion chamber at high-altitude conditions, flash boiling was observed for a liquid oxygen (LOX) jet by De Rosa et al. (2006). Due to a co-flow by gaseous hydrogen, the spray angles, however, were quite narrow for flash boiling sprays. At the same test bench, flash boiling of LOX jets with two injection configurations was investigated and the results were compared to flash boiling sprays of storable fluids (Lamanna et al. 2015). Despite the huge differences in their physical properties, the LOX sprays and the sprays with storable liquids showed a similar spray morphology. The used injection system, however, was limited in terms of controlling and adjusting the injection temperature. In an experimental study by Luo and Haidn (2016) about cryogenic flash boiling, sprays of liquid nitrogen (LN2) for injection times of about $10 \mathrm{~s}$ were observed with high-speed shadowgraphy for different injection conditions and injector geometries. The resulting sprays showed maximum spray angles of about $140^{\circ}$ and the injector geometry and injection pressure did not have big influences on the spray angles. Furthermore, a solidification of nitrogen was observed. Temperature measurements along the spray axis yielded a cool-down of the sprays below the triple point. By Rees et al. (2019b), the validity range of the transition correlations from Cleary et al. (2007) was successfully expanded for the cryogenic fluid LN2 and an asymptotical evolution of the spray angle with increasing superheat was shown. Furthermore, in this study a preliminary PDA measurement campaign showed decreasing droplet diameters with increasing injection temperatures in fully flashing LN2 sprays and a global minimum in the vertical velocity profile as a function of the injection temperature. In a consecutive study by Rees et al. (2019a), the droplet velocity and diameter distributions of highly superheated flash boiling LN2 sprays with constant injection conditions were measured and preliminary results like possible recirculation zones or the existence of a second droplet population were shown.

Because of the very challenging experimental conditions concerning cryogenics as well as the high-altitude atmosphere, there are few experimental data about cryogenic flash boiling and the droplet distributions in the resulting sprays so far. With the following study, we will provide an in-depth insight into the preliminary investigations by Rees et al. (2019a) to generate a comprehensive data base for the validation of numerical models and further numerical investigations about cryogenic flash boiling.

\section{Experimental set-up}

Since the dominating parameters for flash boiling are the injection temperature $T_{\text {inj }}$ and the back pressure $p_{\mathrm{ch}}$, it is important for an experimental investigation to make them adjustable, to keep them constant during the injection period and to make them reproducible. Especially, adjusting and controlling the temperature were partly limited in the few studies with cryogenic flash boiling. This is why we built the new test bench M3.3 with a temperature controlled injection system at DLR Lampoldshausen for a detailed experimental investigation of cryogenic flash boiling processes (Rees et al. 2019a, b).

\subsection{Cryogenic test bench M3.3}

The test bench M3.3 consists of three main systems, as depicted in Fig. 2: the media supply and pressurization system, the cryogenic temperature adjustment and injection system (CTAIS) and the vacuum system. With the first system, all gases (nitrogen, helium, oxygen) for the operation of the test bench are provided and are pressurized with various pressure reducers to the desired pressures. The second of the three main systems consists of a double-walled and vacuuminsulated pressure tank filled with liquid and gaseous nitrogen (GN2), see Fig. 2 on the left and right. By an evacuation or pressurization of the GN2 phase in the pressure tank, the fluid is cooled down or heated up, respectively. In the first case, a new saturation state is reached due to vaporization of a certain amount of LN2. The latent heat of vaporization necessary for this phase change leads to a loss of heat of the liquid/gaseous nitrogen and consequently to a temperature decrease. In the second case, the saturation state after pressurization with GN2 is reached due to condensation of the 

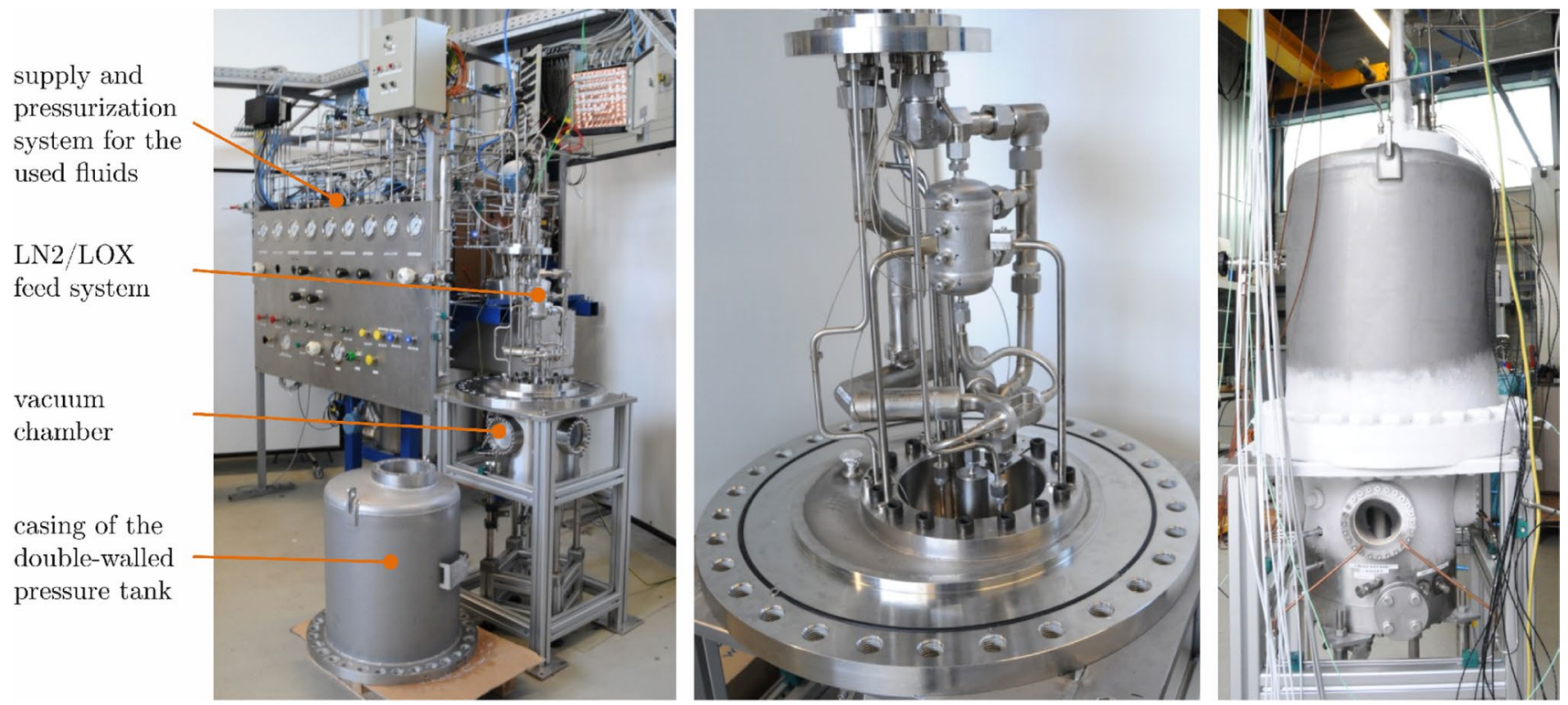

Fig. 2 Test bench M3.3 with supply and pressurization system, open CTAIS and vacuum system (left); open CTAIS with run tank, pneumatic run valve, injector unit and sensors in-between (middle); chilled-down test bench in operation mode (right)

vapor phase. In this process, the latent heat of condensation is released and heats up the nitrogen. Inside the pressure tank is the complete $\mathrm{LN} 2 / \mathrm{LOX}$ feed and injection system, which consists of a $0.5 \mathrm{~L} \mathrm{LN} 2 / \mathrm{LOX}$ run-tank, a mass flowmeter, the injector unit with a pneumatic run valve and the injector nozzle, and piping in-between, see Fig. 2 in the middle. That means that all these sub-systems are completely surrounded by the cooling medium nitrogen to provide a homogeneous temperature distribution from the run-tank to the injector nozzle. Several dynamic pressure and temperature sensors are installed at the nitrogen pressure tank as well as at the feed and injection system to both control and adjust the temperature of the cooling medium and to measure the injection parameters of the jets. The latter is realized by a Pt100A temperature sensor and a dynamic pressure sensor $601 \mathrm{~A}$ by Kistler each installed about $30 \mathrm{~mm}$ upstream of the injector nozzle exit. A hand hole at the top of the pressure tank provides the feedthroughs for the sensors and the supply pipes for LN2, GN2 and Helium. The latter is used to pressurize the pneumatic axial run valve (Axius by Stöhr Armaturen) as well as the cable ducts for the sensor cables inside the pressure tank. The CTAIS is mounted on top of the vacuum system, which is a cylindrical chamber with an inner diameter of $300 \mathrm{~mm}$, a height of $225 \mathrm{~mm}$ from the injector nozzle exit to the bottom of the chamber and four optical accesses with a diameter of $100 \mathrm{~mm}$ each. The four windows are positioned with an angle of $90^{\circ}$ to each other. To prevent icing of the windows due to condensation and freezing of the humidity of the ambient atmosphere, we are running an external window heating system with gaseous nitrogen during the test bench operation. Its copper pipes can be seen at the bottom of the optical access on the right-hand side of Fig. 2. An attached vacuum pump with a pumping speed of $87.5 \mathrm{~m}^{3} / \mathrm{h}$ produces the near-vacuum atmosphere to simulate high-altitude conditions. After evacuating all of the pipes and vessels of the CTAIS the system is chilled-down in about 90 minutes by filling it with LN2. During the chilldown the run-tank is filled with the gaseous test fluid, which is nitrogen for the current study. The test fluid gets liquefied within this chill-down process. The CTAIS allows variable injection conditions, which are summarized in Table 1. In the first run-in tests, we showed that the system is capable of keeping the injection temperature $T_{\mathrm{inj}}$ constant during the whole injection time of about $2 \mathrm{~s}$, that the injection temperature is reproducible in the range of $\pm 0.6 \mathrm{~K}$ for each test run and that the temperature distribution is homogeneous in the whole test fluid feed line (Rees et al. 2019b).

\subsection{Injection conditions}

For further investigation with optical diagnostics, we generated highly superheated flash boiling LN2 sprays, that

Table 1 Possible injection conditions of test bench M3.3

\begin{tabular}{lll}
\hline Parameter & Range & Unit \\
\hline Injection temperature $T_{\text {inj }}$ & $75-120$ & $\mathrm{~K}$ \\
Injection pressure $p_{\text {inj }}$ & $1-20$ & $10^{5} \mathrm{~Pa}$ \\
Back pressure $p_{\mathrm{ch}}$ & $30-1000$ & $10^{2} \mathrm{~Pa}$ \\
Injector diameter $D_{\text {inj }}$ & $1-2$ & $10^{-3} \mathrm{~m}$ \\
Mass flow $\dot{m}$ & $0.08-50$ & $\mathrm{~g} / \mathrm{s}$ \\
\hline
\end{tabular}


are called fully flashing sprays according to the definition of Cleary et al. (2007). Their injection conditions were kept constant and are summarized in Table 2. Such a fully flashing LN2 spray with a degree of superheat of $R_{\mathrm{p}}=69$ and similar injection conditions with $T_{\text {inj }}=90.5 \mathrm{~K}$ and $p_{\text {inj }}=5.5 \times 10^{5} \mathrm{~Pa}$ is depicted in Fig. 3. For all of the generated sprays, we used a single sharp-edged injector with a diameter of $D_{\text {inj }}=1 \mathrm{~mm}$ and a length-to-diameter ratio of $L / D=2.9$. The notable huge tolerance range for the degree of superheat $R_{\mathrm{p}}$ in Table 2 is because of its strong dependence on the back pressure $p_{\text {ch }}$, see Eq. 1. This influence can be nominally circumvented by using the definition of Eq. 2 with the degree of superheat $\Delta T^{*}$. Here, the variations of the back pressure $p_{\text {ch }}$ are normalized by its saturation temperature $T_{\text {sat }}\left(p_{\text {ch }}\right)$. Nevertheless, we will use the definition of $R_{\mathrm{p}}$ in this study due to comparison reasons with literature.

\section{Optical diagnostics}

\subsection{High-speed shadowgraphy}

In a first test campaign, we used high-speed backlight shadowgraphy to visualize the flash boiling LN2 sprays (Rees et al. 2019b). With a xenon light source the sprays were illuminated from the backside through one of the four optical accesses of the vacuum chamber. A translucent milk glass screen was placed between the light source and the chamber window to provide a homogeneous background. The highspeed camera was positioned on the opposite optical access

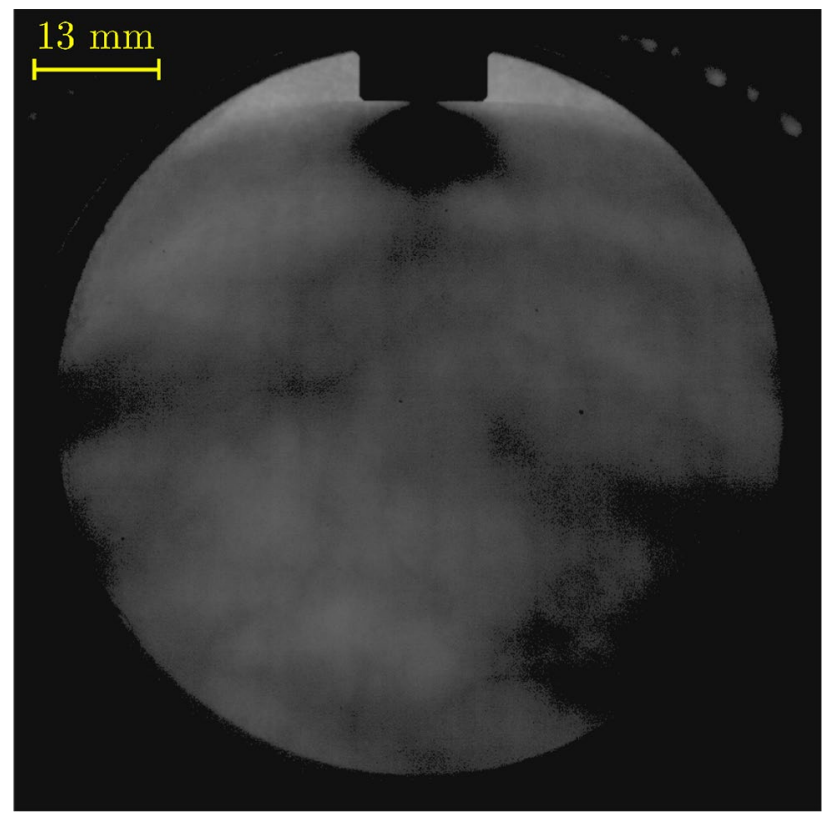

Fig. 3 Highly superheated fully flashing LN2 spray after $120 \mathrm{~ms}$ at $R_{\mathrm{p}}=69\left(T_{\text {inj }}=90.5 \mathrm{~K}, p_{\text {inj }}=5.5 \times 10^{5} \mathrm{~Pa}\right.$ and $\left.p_{\text {ch }}=55 \times 10^{2} \mathrm{~Pa}\right)$
Table 2 Injection conditions of the used LN2 sprays

\begin{tabular}{lll}
\hline Parameter & Value & Tolerance \\
\hline Inject. temp. $T_{\text {inj }}$ & $89.7 \mathrm{~K}$ & $\pm 0.6 \mathrm{~K}$ \\
Inject. pressure $p_{\text {inj }}$ & $4.4 \times 10^{5} \mathrm{~Pa}$ & $\pm 0.4 \times 10^{5} \mathrm{~Pa}$ \\
Back pressure $p_{\text {ch }}$ & $73 \times 10^{2} \mathrm{~Pa}$ & $\pm 27 \times 10^{2} \mathrm{~Pa}$ \\
Inject. diameter $D_{\text {inj }}$ & $1 \times 10^{-3} \mathrm{~m}$ & $\pm 0.05 \times 10^{-3} \mathrm{~m}$ \\
Length-to-diameter $L / D$ & 2.9 & \pm 0.2 \\
Inject. velocity $u_{\text {inj }}$ & $18.1 \mathrm{~m} / \mathrm{s}$ & $\pm 3 \mathrm{~m} / \mathrm{s}$ \\
Chamber wall temp. $T_{\mathrm{w}}$ & $196.6 \mathrm{~K}$ & $\pm 3.0 \mathrm{~K}$ \\
Degree of superh. $R_{\mathrm{p}}$ & 60 & \pm 30 \\
Degree of superh. $\Delta T^{*}$ & 0.91 & \pm 0.04 \\
Weber number $W e$ & 21 & \pm 10 \\
Jakob number $J a$ & 746 & \pm 395 \\
Reynolds number $R e$ & 131,952 & $\pm 22,165$ \\
\hline
\end{tabular}

of the chamber and the camera lens was focussed on the injector plane. The optical set-up is shown schematically in Fig. 4. The high-speed camera was set to a framerate of $10,000 \mathrm{fps}$, an exposure time of $97 \mu \mathrm{s}$ and a frame size of $1024 \times 1024$ pixels.

\subsection{Phase Doppler system}

\subsubsection{General discription of the used system}

After a preliminary PDA campaign in the dual-mode configuration presented by Rees et al. (2019a), the Dantec PDA system was set up in the 2D fibre configuration. The optical set-up is depicted in Figs. 5 and 6 (left) and the main components of the PDA system are summarized in Table 3. Because of the geometrical limited optical access to the spray, we tilted the transmitter and receiver probes and positioned them with an off-axis angle of $\varphi=7^{\circ}$ and $5^{\circ}$, respectively, to use forward scattering first order refraction at a total off-axis angle of $\varphi=12^{\circ}$. Tilting both probes and choosing the total off-axis angle of $\varphi=12^{\circ}$ provides an optimal compromise between the geometrical field of view and the dominant refraction mode. The field of view determines the matrix of measurement positions, which are shown in Fig. 6 on the right. We set both DPSS lasers to a power of about $40 \mathrm{~mW}$ which results

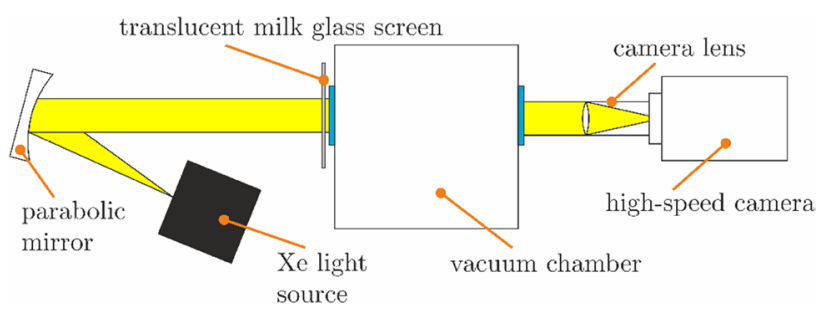

Fig. 4 Scheme of the optical set-up for high-speed backlight shadowgraphy at test bench M3.3 
Table 3 Components of the optical PDA set-up

\begin{tabular}{ll}
\hline Component & Specification \\
\hline 2 DopplerPower DPSS lasers & $1 \mathrm{~W} ; 488 \mathrm{~nm}$ and $514 \mathrm{~nm}$ \\
2D transmitter probe & $D=60 \mathrm{~mm} ; \varphi=7^{\circ}$ \\
2D fibre PDA receiver probe & $D=112 \mathrm{~mm} ; \varphi=5^{\circ}$ \\
Two front lenses & $f=500 \mathrm{~mm}$ \\
Burst processor P800-2D & \\
2D traverse system & \\
\hline
\end{tabular}

in a power of approximately $9 \mathrm{~mW}$ for each laser beam in the measurement volume. A spatial filter with a slit of $100 \mu \mathrm{m}$ was chosen. The PDA system was first adjusted and aligned with the help of a water spray generated by a similar nozzle like the one used in the CTAIS. We placed a calibrated needle with a diameter of $1 \mathrm{~mm}$ into the injector nozzle of the CTAIS and illuminated it by one of the laser beams to align the traverse system with the mounted transmitter and receiver probes horizontally. For its vertical alignment, we illuminated the lower edge of the injector nozzle with the laser. The refractive index for nitrogen was set to $n=1.205$ according to an interpolation of values derived from Johns and Wilhelm (1937) with respect to the used wavelengths and to the temperature range for the LN2 measurement campaign. With this PDA system, we measured the vertical and horizontal droplet velocity as well as the droplet size distributions at different radial and axial locations in fully flashing LN2 sprays at constant injection conditions. Based on the optical set-up of the PDA system, droplets with a maximum particle diameter of $83.2 \mu \mathrm{m}$ can be measured.

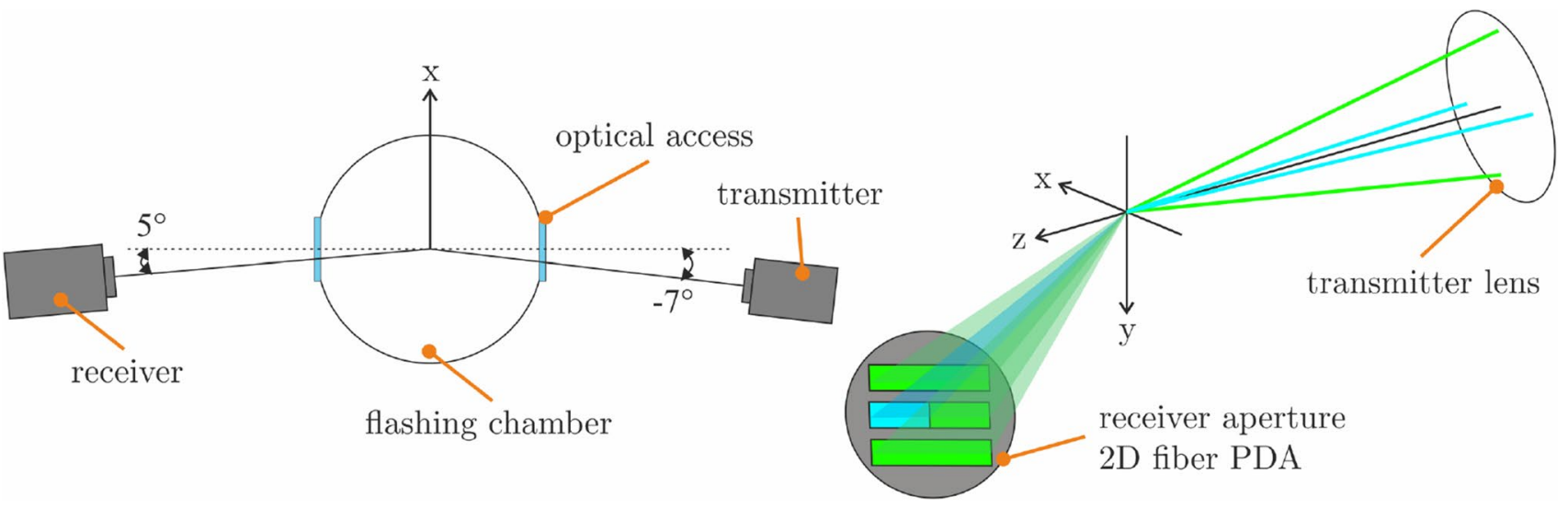

Fig. 5 Top view of the optical set-up of the 2D Fibre PDA system centered on the injector symmetry axis at test bench M3.3 (left) and receiver aperture of the used 2D fibre configuration (right)
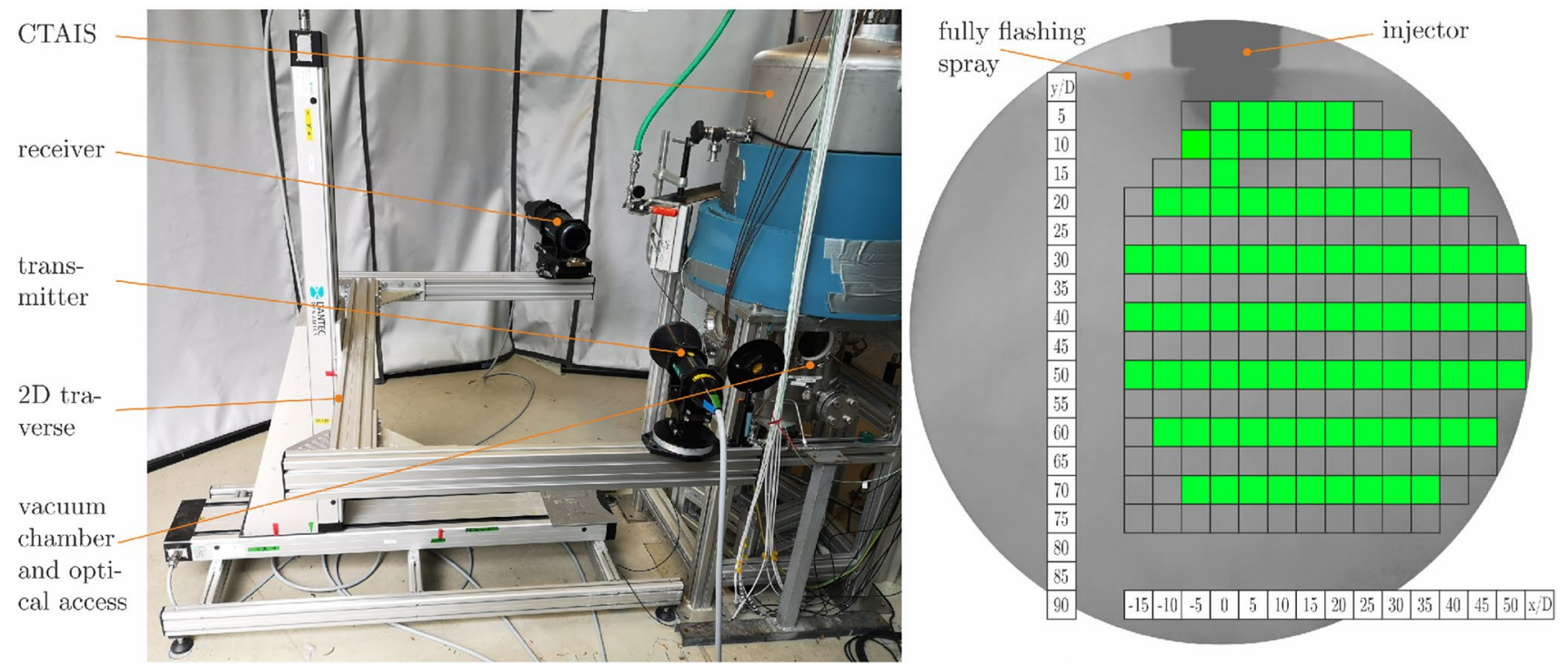

Fig. 6 2D Fibre PDA system at test bench M3.3 (left) and measurement positions in a fully flashing LN2 spray (right) 
We set the velocity ranges to $-67 \mathrm{~m} / \mathrm{s} \leq U \leq 203 \mathrm{~m} / \mathrm{s}$ for the vertical velocity component and to $-160 \mathrm{~m} / \mathrm{s} \leq V \leq 241 \mathrm{~m} / \mathrm{s}$ for the horizontal one.

For each position of the PDA position matrix on the righthand side in Fig. 6, one single flash boiling LN2 spray was generated and the data were recorded for a duration of 12 s. Data from different positions are thus related to different injection events, where shot-to-shot variations can not be excluded. The start of the data recording is synchronized by the valve opening signal prior to the injection. We evaluated and arithmetically averaged the detected signals within a time period of $t=120-220 \mathrm{~ms}$ after injection start due to steady state injection conditions in this interval.

\subsubsection{Error estimation of the PDA measurements}

A major drawback of the small off-axis angle of $\varphi=12^{\circ}$ is the dwindling dominance of the scattering light by first order refraction compared to light scattered by Fraunhofer diffraction. This can cause an interference of the two kinds of scattering light especially for smaller droplets below $10 \mu \mathrm{m}$ in diameter and can lead to a biased size determination for droplets of this size class. Hence, using an off-axis angle of $\varphi=12^{\circ}$ induces a systematical error for the droplet size determination. To quantify this measurement error, we performed two PDA measurement series in a water spray at ambient conditions and with a similar injector geometry as for the LN2 campaign. Between the two test series, we changed the off-axis angle from $\varphi=12^{\circ}$ to $\varphi=30^{\circ}$. All the other set-up and spray conditions were kept constant except for the power of the laser, which was increased from $9 \mathrm{~mW}$ at $\varphi=12^{\circ}$ to about $22 \mathrm{~mW}$ at $\varphi=30^{\circ}$ for each laser beam. The higher value of $\varphi=30^{\circ}$ is an off-axis angle commonly used for water sprays, like e.g. by Tropea et al. (1996). Comparing the results of both test series yielded an averaged deviation of about $\pm 26 \%$ for the set-up with $\varphi=12^{\circ}$ compared to the one at $\varphi=30^{\circ}$. Therefore, we can quantify the reliance of the used PDA set-up with an off-axis angle of $\varphi=12^{\circ}$ by a systematical measurement error of $\pm 26 \%$ for the size determination. Besides this systematical error, the maximum statistical errors can be quantified with $\Delta U= \pm 6.1 \mathrm{~m} / \mathrm{s}, \Delta V= \pm 8.0 \mathrm{~m} / \mathrm{s}$ and $\Delta D= \pm 3.3 \mu \mathrm{m}$ and occur mainly at measurement positions close to the nozzle exit. For reasons of clarity in Figs. 11, 13 and 18 only the statistical errors of the droplet size determination are depicted as error bars.

\section{Results and discussion}

With the described PDA set-up, we measured simultaneously the local vertical and horizontal velocity components of the droplets as well as their diameters in one single injection event. The corresponding results of each quantity or rather their local distributions are shown and discussed in the following subsections.

\subsection{Vertical velocity distribution}

The spatial distribution of the arithmetic mean velocity component $U$ as a function of the dimensionless radial distance from the injector axis $x / D$ is shown in Fig. 7 on the left-hand side. Depending on the measurement position 10-3000 Doppler-bursts, i.e. detected droplets, were detected by the burst processor and were used for evaluation. Their local distribution is depicted on the right-hand side of Fig. 7. At different axial distances $y / D$, each lateral distribution is mapped by a color code. Contrary to all the other radial distances, at $y / D=15$ the only single position measured was that on the spray axis, i.e. at $x / D=0$. Since the measured vertical and horizontal velocities at this position are very close to the values at $y / D=10$ and $y / D=20$, they are difficult to see in Figs. 7, 9, 16 and 17.

At positions $10 \leq y / D \leq 20$ and $-10 \leq x / D \leq 10$ close to the injector orifice the vertical velocities with $U=50-70 \frac{\mathrm{m}}{\mathrm{s}}$ are much higher than the injection velocity $u_{\text {inj }}=18.1 \frac{\mathrm{m}}{\mathrm{s}}$, which is calculated from the arithmetical mean of the measured liquid's mass flow rate within the evaluation period. Even closer to the injector at $y / D=5$, smaller velocities than slightly downstream were measured. However, the number of detected droplets is very low in the near-nozzle region (see Fig. 7 on the right-hand side). On one hand, the low data rate is caused by the optical dense core region of the spray, where most of the scattering light of the droplets is not reaching the PDA receiver probe. On the other hand, a lot of the existing droplets in these core regions are non-spherical structures or ligaments, whose Doppler signals are rejected by the measurement system. As already described by Lecourt and Barricau (2009), Cleary et al. (2007) and Yildiz et al. (2004) in experimental flash boiling studies with storable fluids, optical diagnostic methods like PDA reach their limits in those kinds of spray regions. In the upper outer region of the spray at $y / D \leq 20$, the vertical velocity $U$ is strongly decreasing with increasing radial distance due to a distinctive horizontal momentum direction for the widening of the spray in that area. This strong variation in the velocity magnitude is due to the complex interplay between the gas dynamics and non-equilibrium fluid transition. At high superheat levels, nucleation and bubble growth can occur already within the nozzle. This leads to a drastic reduction in the speed of sound for the twophase flow and the nozzle flow becomes choked. Consequently, the two-phase jet becomes under-expanded and experiences a Prandtl-Meyer expansion flow at the nozzle exit (Lamanna et al. 2014; Kurschat et al. 1992). The 

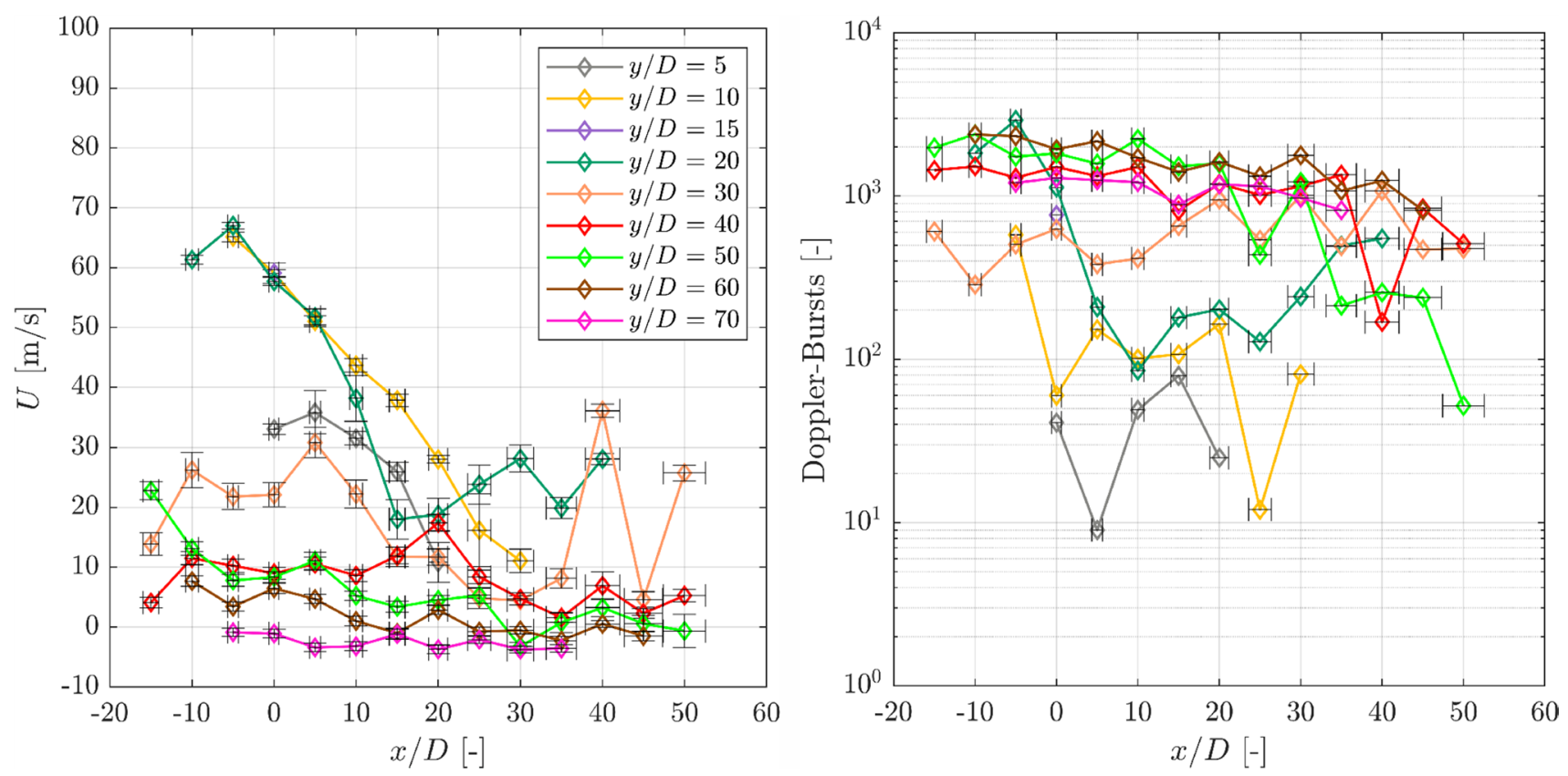

Fig. 7 Spatial distributions of the mean vertical velocity $U$ (left) and of the number of valid Doppler-bursts (right) in fully flashing LN2 sprays; injection conditions see Table 2

Prandtl-Meyer expansion fans are responsible for the strong variation of the velocity magnitude between the axis with a cumulative effect of multiple characteristic waves and the edges, where only a few characteristic waves accelerate the jet locally. Further downstream at $y / D \geq 30$ considerably smaller vertical velocities were measured with a less steep decline towards the lateral spray edges. After passing the Mach disc, the profiles of the vertical velocity $U$ get flat due to the strong deceleration caused by the normal shock uniformly in the horizontal direction. These trends are consistent with the profiles of the horizontal velocity $V$ on the left-hand side of Fig. 9. Indeed, for $y / D>40$, the velocity profiles get quite homogeneous in the horizontal direction due to the decelerating action of the normal shock of the Prandtl-Meyer expansion flow system. In addition, the velocity oscillations at $y / D=30$ and $x / D>30$ are due to the interaction of the flow with the oblique shock, emanating from the Mach disc. Simultaneously, the number of detected droplets increases to values above 1000 with resulting mean validation rates of $70 \%$. The evolution of the vertical velocity profiles shows, that internal energy of the LN2 jet is transformed into kinetic one immediately downstream of the injector orifice. Here, the highest vertical velocities were measured due to spray expansion. Local peaks in the velocity profiles like at position $x / D=40$ and $y / D \leq 30$ may also occur because of local asymmetries or density fields, which can be seen, e.g., in the lower right corner of Fig. 3. Another possible reason could be small shot-to-shot variations between each test run and measurement position, respectively.

In Fig. 8, the vertical velocity profile along the spray axis $U_{\text {axis }}$ of the LN2 sprays $(x / D=0)$ is depicted and compared with two flash boiling ethanol sprays with a degree of superheat of $R_{\mathrm{p}}=11.7$ and $R_{\mathrm{p}}=63.7$ by Kamoun et al. (2012) and Kamoun (2015), respectively. With $p_{\mathrm{ch}}=60 \times 10^{2} \mathrm{~Pa}$ the chamber pressure of both ethanol sprays is similar to the one of the LN2 vacuum chamber in this study. Due to the different chamber temperatures (for the experiments of Kamoun et al. $T_{\text {ch }} \approx 291 \mathrm{~K}$, in this work $T_{\text {ch }} \approx 197 \mathrm{~K}$ ), however, the gas density $\rho_{\mathrm{ch}}$ in the chamber will be different, thus resulting in different air entrainment rates, see Eq. 3.

The initially high LN2 axial velocities of about $U_{\text {axis }} \approx 60 \frac{\mathrm{m}}{\mathrm{s}}$ are very close to the velocities of the ethanol sprays and are believed to occur due to the evaporation of ligaments and big liquid structures and the subsequent violent expansion of the resulting smaller droplets. The strong similarity of the LN2 and the ethanol data close to the injector plane at $10<y / D<20$ is due to the still low entrainment rates for both liquids in that spray region, see Eq. 3. Further downstream at $20<y / D<40$, the axial velocity strongly decreases because of the increasing surrounding gaseous nitrogen entrainment with a decelerating effect on the secondary droplets. However, the deceleration of LN2 is much stronger than for the ethanol sprays. First, this difference can be explained by considering the larger entrainment rate of the LN2 jets which leads to a higher axial deceleration 


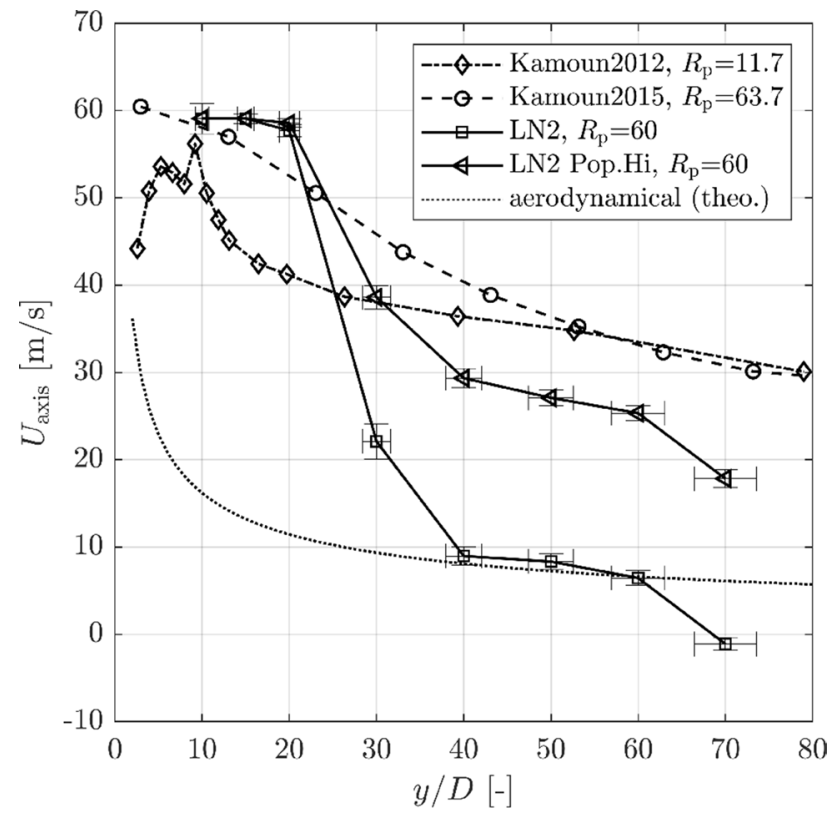

Fig. 8 Axial velocity $U_{\text {axis }}$ profile along the spray axis $y$ at $x / D=0$ of LN2 sprays compared with two flash boiling ethanol sprays by Kamoun et al. (2012) $\left(R_{\mathrm{p}}=11.7 ; p_{\mathrm{ch}}=60 \times 10^{2} \mathrm{~Pa}\right)$ and Kamoun (2015) $\left(R_{\mathrm{p}}=63.7 ; p_{\mathrm{ch}}=60 \times 10^{2} \mathrm{~Pa}\right) ; \mathrm{LN} 2$ injection conditions see Table 2

for the large size droplets in LN2 than for ethanol. Indeed, according to Medrano et al. (2017) and reference therein, the entrainment rate $\dot{m}_{\mathrm{e}}$ can be expressed as

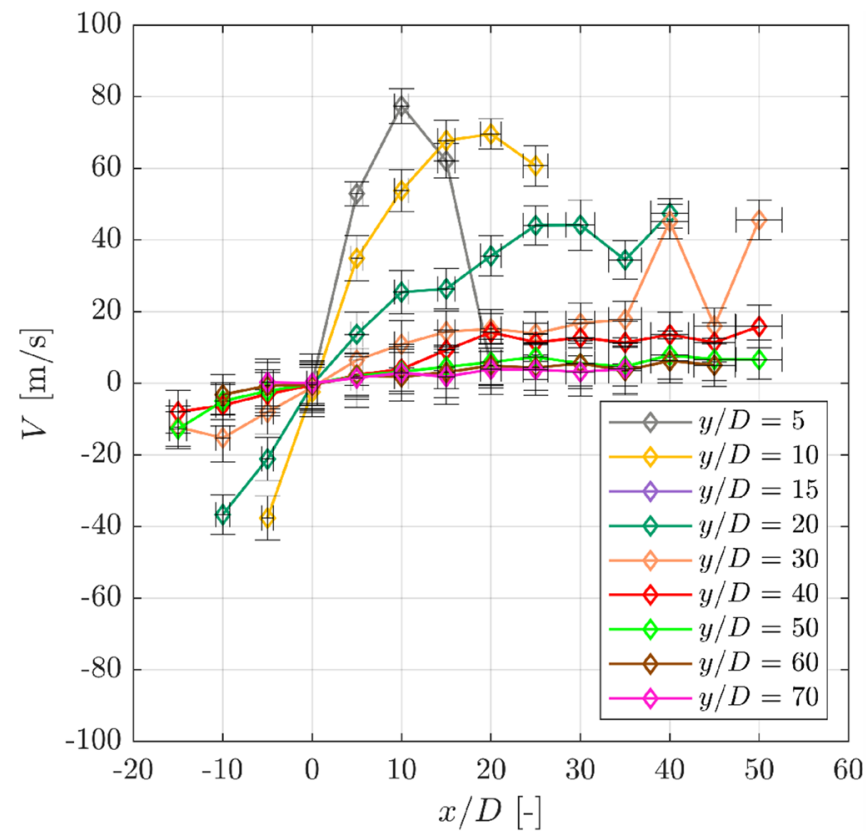

$\dot{m}_{\mathrm{e}}=K_{\mathrm{e}} \frac{y}{D}\left(\frac{\rho_{\text {ch }}}{\rho_{\text {fluid }}}\right)^{0,5}$

with the entrainment rate coefficient $K_{\mathrm{e}}$ and the fluid density $\rho_{\text {fluid }}$. Due to the colder operating vacuum chamber in the LN2 experiments, it is clear why they experience a higher entrainment rate and, therefore, a larger deceleration. Second, the difference can be explained by taking into account the characteristics of the velocity measurements obtained with the Laser Correlation Velocimetry technique (LCV), as done in the study of Kamoun (2015). The LCV technique detects the extinction signal, caused by the passage of droplets in the measurement volume. For a droplet ensemble, the LCV technique is therefore more sensitive to the larger droplet sizes, since they cause the highest extinction signal. This statement is also corroborated in Fig. 8, where the PDA velocity measurements for the Hi-population are much closer to the LCV data of ethanol than the curve of the total LN2 droplet ensemble (refer to Sect. 4.4 for information about the different droplet populations). This occurs because larger droplets exhibit a larger inertia and, therefore, conserve their momentum longer in time. The opposite occurs for the Lo-population droplets: due to their reduced inertia, they decelerate rapidly and eventually follow the gas flow. Indeed, for $y / D \geq 40$, the measured axial velocities $U_{\text {axis }}$ are close to the theoretical relation

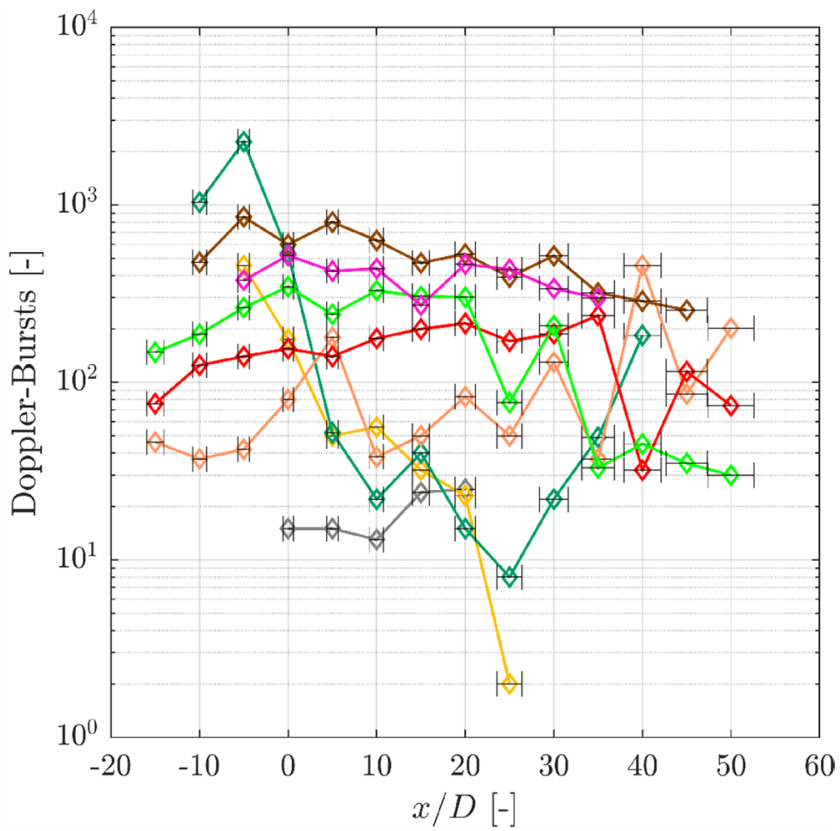

Fig. 9 Spatial distributions of the mean horizontal velocity $V$ (left) and of the number of valid Doppler-bursts (right) in fully flashing LN2 sprays; injection conditions see Table 2 


$$
\frac{U_{\text {axis }}}{u_{\text {inj }}}=\frac{1.2}{\sqrt{0.186 \frac{y}{D}}}
$$

by Abramovich (1963), however, with parameters established for atmospheric conditions. An overview for the appropriate choice of the coefficients can be found in the study by Franquet et al. (2015). The authors showed that the hyperbolic decay found for submerged incompressible jets holds also for under-expanded jets, provided that the variables are scaled through the notional nozzle approach. Failing to correctly compute this equivalent thermodynamic state leads inevitably to a large discrepancy in the modeling of the self-similar law. The existence of self-similar velocity profiles for flash-boiling jets has been demonstrated by several authors, like e.g. by Kamoun et al. (2012), Kamoun (2015) or within the detailed overview of Witlox and Bowen (2002). Neither the LCV nor the PDA velocity measurements provide enough information to correctly calculate the equivalent thermodynamic state in the notional nozzle approach. Therefore, we are using the simplified form of Eq. 4 to demonstrate the hyperbolic decay in the far field. Indeed, although not properly scaled, all experiments at $R_{\mathrm{p}} \approx 60$ are basically parallel in the far field region, see Fig. 8.

The close distance between the measured axial velocities $U_{\text {axis }}$ and the theoretical relation according to Eq. 4 implies that the smaller droplets follow completely the gaseous flow. At the position $y / D=70$ the vertical velocity $U_{\text {axis }}$ drops below the theoretical curve according to Eq. 4 and gets even negative. The comparison with the left-hand side of Fig. 7 reveals that the whole radial profile at $y / D=70=$ const. is characterized by negative vertical velocities. These negative velocities indicate the existence of recirculation zones due to wall interaction and/or especially shock systems in the fully flashing LN2 sprays. As a consequence, the creation of vapor-rich areas due to recirculation or compression downstream of these shocks can cause local supersaturation which leads to re-condensation of the nitrogen vapor. Indications of those kind of recirculation zones can be found e.g. as dark structures within the LN2 shadowgraph image in Fig. 3 as well as in shadowgraph images of fully flashing LN2 sprays by Rees et al. (2019a) and of iso-octane sprays by Lamanna et al. (2014). The sudden decay in the axial velocity of the LN2 experiments at $y / D=70$ was not observed in the ethanol sprays. This is due to the occurrence of a re-circulation zone in the LN2 jets, as corroborated by the numerical simulations of Gärtner et al. (2020). In their numerical study with experimental data of both fluids, the presence of negative velocity fields due to shock systems in the same regions was shown. So deviations from the far-field hyperbolic decay are observed only in presence of the re-circulation zones and may cause even negative velocities, as shown in Fig. 7 on the left-hand side.

\subsection{Horizontal velocity distribution}

With the second pair of laser beams, which have a wavelength of $488 \mathrm{~nm}$, we measured the horizontal velocity component $V$. Their spatial distribution is shown on the left-hand side in Fig. 9. On the right-hand side of the figure, the local distribution of the related number of detected droplets is depicted again. The reason for the different numbers of the local Doppler-bursts for the horizontal velocity measurements compared to the droplet number of the vertical ones on the right-hand side of Fig. 7 is that the signals for the horizontal velocity components are derived by the second laser system with the wavelength of $\lambda=488 \mathrm{~nm}$ instead of the vertical one with $\lambda=514 \mathrm{~nm}$, see the right-hand side of Fig. 5. The mean validation rate for the horizontal velocity measurement is about $66 \%$.

Since a vertical and rotationally symmetrical spray has no horizontal velocity components on its axis of symmetry, all of the horizontal velocity profiles are running through the coordinate origin and are point symmetric to it. Both features are visible on the left-hand side of Fig. 9. Immediately adjacent to the spray axis and with $y / D=5$ very close to the injector the horizontal velocities are increasing strongly to peak values up to $V=80 \frac{\mathrm{m}}{\mathrm{s}}$. With growing distances from the injector like $10 \leq y / D \stackrel{\mathrm{s}}{\leq} 20$, the maximum peaks in the profiles are getting lower and their radial positions are shifted towards the spray outer edges. This means that the evolution of the radial horizontal velocity profiles is contrary compared to the one of the vertical velocities. Hence, the transformation of internal energy of the bulk liquid into kinetic one occurs after a certain radial distance from the injector axis predominantly into the lateral direction. The decline of the horizontal velocity profiles for $y / D \approx 5-20$ after the respective maximum peak is caused by two issues: First, in this region the number of validated droplets with values in the order of magnitude of about 10 is significantly low compared to other regions of the spray, see the local distribution on the right-hand side of Fig. 9. A possible reason for the low data rate is the limited optical access induced by geometrical constraints like the strong curvature of the window margin in the near-nozzle region. Interactions of both, the illuminating laser beams and the scattering light, with the window margins can impede the complete droplet illumination and the entire collection of the scattering light, respectively. The incomplete light paths can cause wrong velocity calculations in turn. With proper alignment of the optical PDA components prior to the measurements and a suitable choice of the measurement position matrix according to Fig. 6 on the right-hand side, we minimized these influences of the window margins. Second, the low data rate can be caused by the so-called barrel shock as part of typical shock systems, like they were observed multiple times in strongly superheated storable fluids and the resulting fully 


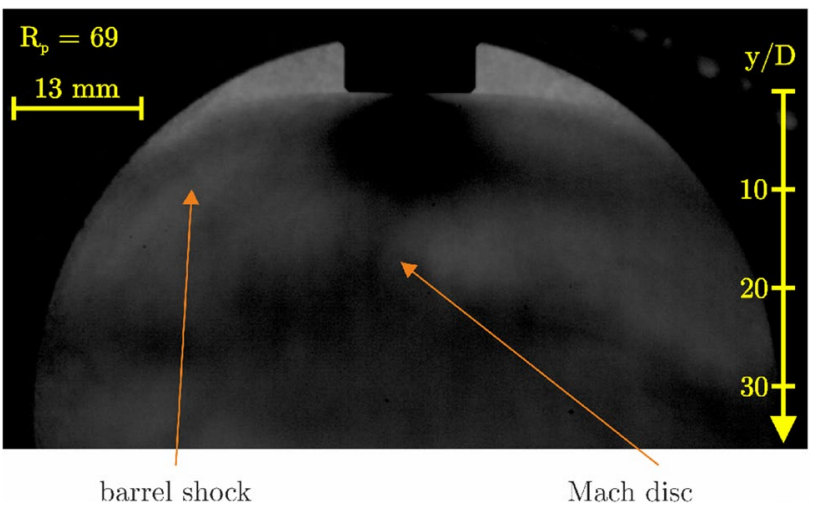

Fig. 10 Shock structures in a highly superheated fully flashing LN2 spray after $120 \mathrm{~ms}$ at $R_{\mathrm{p}}=69$ with Mach disc and barrel shock $\left(T_{\text {inj }}=90.5 \mathrm{~K}, p_{\text {inj }}=5.5 \times 10^{5} \mathrm{~Pa}\right.$ and $\left.p_{\text {ch }}=55 \times 10^{2} \mathrm{~Pa}\right)$

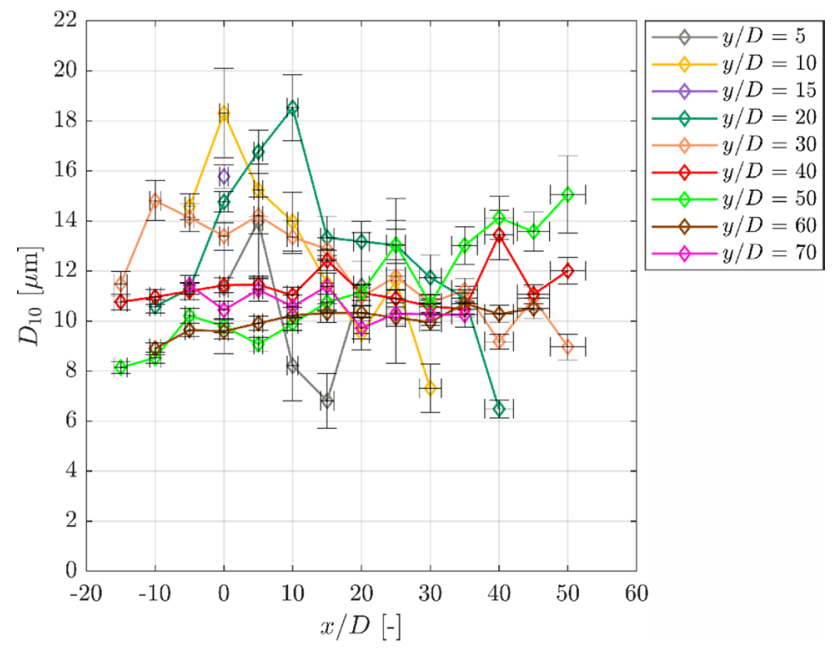

Fig. 11 Spatial distributions of the arithmetic mean diameter $\mathrm{AMD}=D_{10}$ in fully flashing LN2 sprays; injection conditions see Table 2

flashing sprays (Kurschat et al. 1992; Simões Moreira et al. 2002; Lamanna et al. 2014). As visualized in Fig. 10 with the help of the shadowgraph image of a fully flashing LN2 spray with a degree of superheat of $R_{\mathrm{p}}=69\left(T_{\mathrm{inj}}=90.5 \mathrm{~K}\right.$, $p_{\text {inj }}=5.5 \times 10^{5} \mathrm{~Pa}$ and $p_{\text {ch }}=55 \times 10^{2} \mathrm{~Pa}$ ) this barrel shock lies in the same spray region where the decline of the horizontal velocity is observed.

With growing axial distances $y / D$ from the injector orifice the horizontal velocities are decreasing strongly and the lateral increase of each profile is getting less steep until they stay almost constant from $x / D=20$ onwards. For $y / D \geq 50$, the velocity profiles become almost congruent and low mean values about $V=5 \frac{\mathrm{m}}{\mathrm{s}}=$ const. are reached in the outer regions of the spray. Hence, the growing influence of gravity on the droplets in the spray causes droplet trajectories similar to a ballistic trajectory, where the horizontal velocity component decreases in favor of the vertical one. A similar radial velocity evolution in flash boiling ethanol sprays was observed by Kamoun et al. (2012).

\subsection{Diameter distribution}

The data for the determination of the droplet diameters are derived from the laser system with the wavelength of $514 \mathrm{~nm}$ and the accompanying three detectors, see Fig. 5 on the right-hand side. Thus, the diameter measurements are based on the same signals used for the vertical velocity components $U$ depicted on the right-hand side of Fig. 7 . However, the number of valid diameters is reduced by a factor of about 0.84 because of a mean spherical validation rate of $84 \%$, which is a stricter validation criterion than the ones used for velocity measurements alone. The respective spatial distribution of the resulting arithmetic mean diameter (AMD, or $D_{10}$ ) is depicted in Fig. 11. At the closest position to the injector with $y / D=5$ the droplet diameters seem to be quite low with no clear trend. As already mentioned, the validation rates are very low in this region due to the large amount of ligaments and non-spherical particles, which is why especially the diameter determination gets biased that close to the injector. The presence of such particles in those dense core regions close to the injector orifice and the accompanying problems for optical diagnostic methods is well-known in literature and was observed by Yildiz et al. (2004), Cleary et al. (2007) or Lecourt and Barricau (2009), for example. Downstream in the region $10 \leq y / D \leq 30$ AMDs of $14-18 \mu \mathrm{m}$ were measured close to the injector axis. Laterally, they decrease to values below $D_{10}=10 \mu \mathrm{m}$. Further downstream the radial diameter profiles are getting smoother with droplet diameters of $D_{10}=8-10 \mu \mathrm{m}$. However, towards the periphery of the spray for $x / D>30$ the diameter profiles are getting unsteady again with even an increasing progression for the profiles at $y / D=40$ and 50. Consequently, the ligaments and non-spherical particles close to the injector continuously disintegrate with growing axial distance to smaller droplets until an almost monodisperse droplet distribution at $y / D \approx 40$ is reached in the spray.

The increase in diameter at the positions $x / D>30$ and $y / D=40$ and 50 can be caused by recirculation flows close to the window margins, like e.g. the dark density fields in the lower right corner of Fig. 3. Furthermore, this is proved by the sharp decline of the data rate at these measurement positions according to Fig. 7 on the right-hand side. Increasing droplet diameters at the spray edges of flash boiling water and Freon-11 jets were observed by Brown and York (1962), too.

Another distinctive feature is the location of the radial profile at $y / D=70$, which lies above the ones at $y / D=50$ and 60 close to the spay axis. This region is marked with a yellow box within the shadowgraph images 
of a fully flashing LN2 spray at $R_{\mathrm{p}}=191.4 \mathrm{in} \mathrm{Fig.} \mathrm{12.} \mathrm{The}$ time increment between each shadowgraph frame is 0.1 ms. Within the yellow boxes, upstream flowing liquid structures are visible, that are bigger than the fine spray droplets in the surroundings. These liquid structures consist of secondary droplets, which are chilled-down to thermodynamic equilibrium and originate from below the optical accesses. By collision with droplets of the main flow and the corresponding interaction, the secondary droplets are decelerated in their upstream motion. Depending on the relative velocities as well as the direction and position of each collision event, the interaction can be droplet coalescence or further break-up into smaller satellite droplets (Ashgriz and Poo 1990).
On the left-hand side in Fig. 13, the AMD profile along the spray axis $D_{10 \text {,axis }}$ of the LN2 sprays $(x / D=0)$ is depicted and compared with a flash boiling spray of the refrigerant R-134A with a degree of superheat of $R_{\mathrm{p}}=6.6$ by Yildiz et al. (2004). For comparison reasons, we normalized the averaged diameters with the injector diameter $D_{\text {inj. }}$ Obviously, the AMDs of both spray types are in the same order of magnitude with $10 \mu \mathrm{m}<D_{10 \text {,axis }}<20 \mu \mathrm{m}$ despite the different fluids and degrees of superheat. The slight diameter increase at $y / D=5$ for both sprays is presumably not because of thermodynamical or aerodynamical reasons but more likely due to the limitations of the optical diagnostics in the near-injector region with non-spherical liquid structures. Further downstream in the LN2 sprays the AMDs
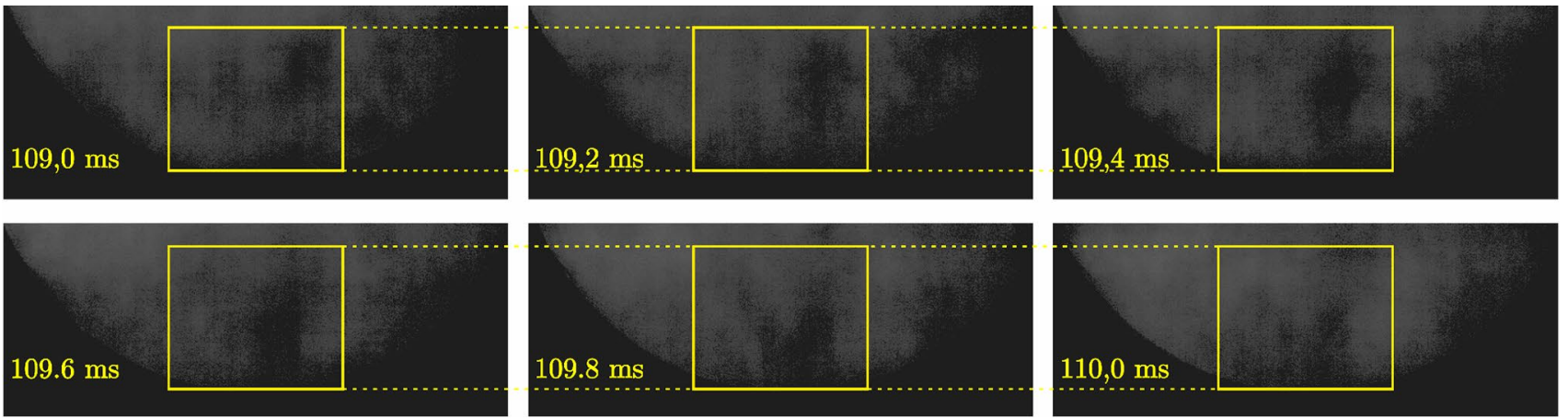

Fig. 12 Recirculation zones at $y / D=70$ in shadowgraph images of a fully flashing LN2 spray at $R_{\mathrm{p}}=69$; time increment $0.2 \mathrm{~ms}$ between each frame
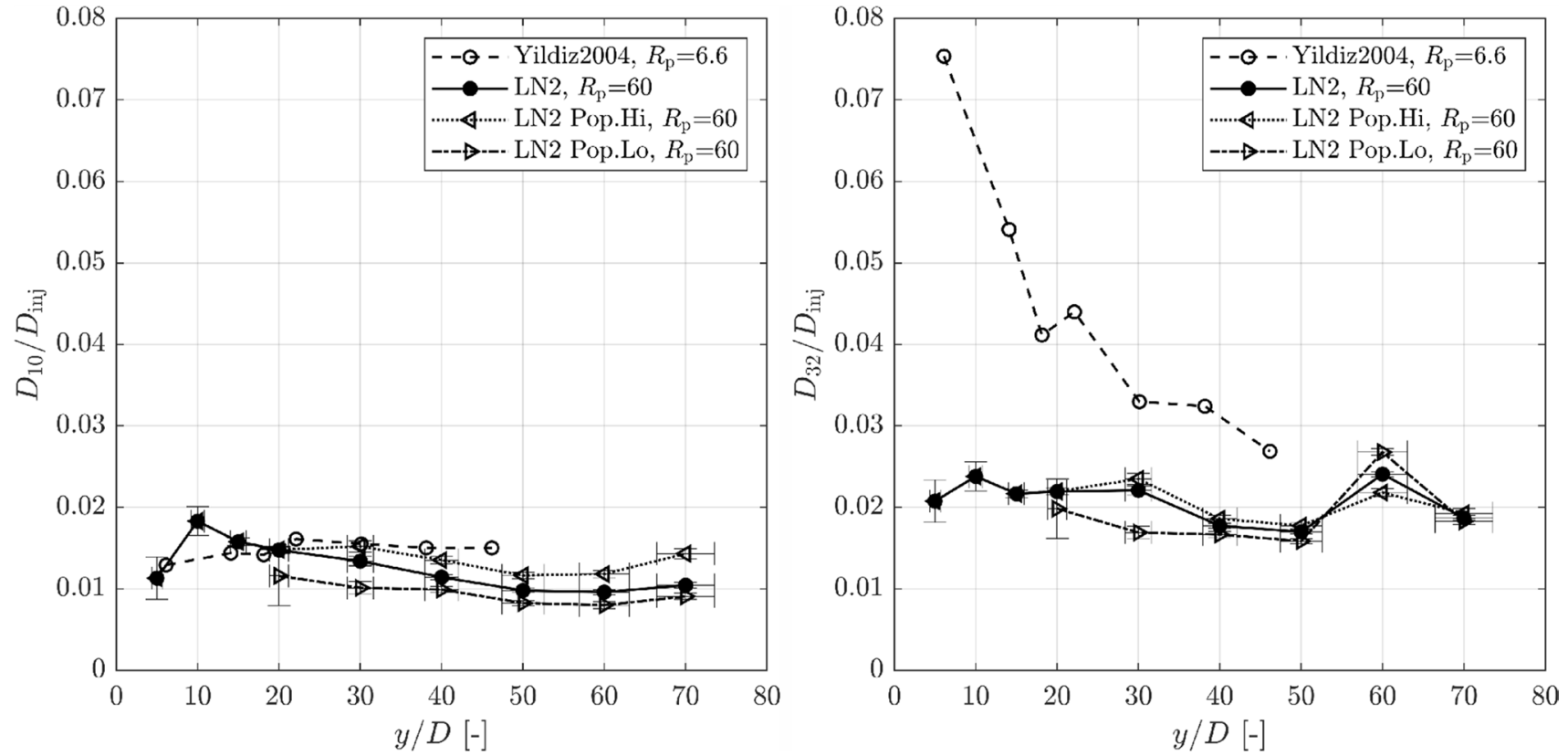

Fig. 13 Axial diameter profile $D_{10 \text {,axis }}$ (left) and $D_{32 \text {,axis }}$ (right) at $x / D=0$ of LN2 sprays compared with a R-134A spray at $R_{\mathrm{p}}=6.6$ by Yildiz et al. (2004); $D_{\text {inj,LN2 }}=1 \times 10^{-3} \mathrm{~m}$ and $D_{\text {inj,Yildiz }}=5 \times 10^{-3} \mathrm{~m}$; further LN2 injection conditions see Table 2 
decrease along the spay axis, whereas they stay almost constant in R-134A. The greater decline of the droplet AMDs in the LN2 sprays is due to their considerably higher degree of superheat, which is roughly by a factor of ten, and the resulting stronger atomization by flash evaporation. The huge difference between the degrees of superheat of both spray types is even more obvious regarding the axial profiles of the respective Sauter mean diameters (SMD) $D_{32 \text {,axis }}$, that are shown on the right-hand side of Fig. 13. For the positions $5<y / D \leq 15$ close to the injector orifice, the axial SMDs of R-134A are about three to four times higher than for LN2. While the axial SMD profile of LN2 decreases only slightly, the decline for R-134A is very sharp and reaches similar values only after a distance of at least $y / D \geq 50$. The high degree of superheat in the LN2 sprays triggers the jet break-up already in the injector or directly at its orifice and atomizes the jet into much smaller droplets than the less superheated R-134A. The short distance between the AMD and the SMD profile of the LN2 sprays shows the ability of highly superheated jets to generate an almost monodisperse spray pattern. The significant increase around the position $y / D \approx 60$ shows again the backflow as outlined above.

\subsection{Droplet populations}

As already shown in our preliminary analysis of PDA measurements in flash boiling LN2, two different classes of droplets were found in the sprays as depicted in Fig. 14 exemplarily at the spray position $x / D=0$ and $y / D=30$ (Rees et al. 2019a). They are differing mainly in their mean vertical velocity $U$, why we chose a velocity threshold of $U_{\text {thresh }}=5 \mathrm{~m} / \mathrm{s}$ to separate the entire droplet population into a Hi-population with velocities $U>U_{\text {thresh }}$ and a Lo-population with $U<U_{\text {thresh }}$, respectively. The velocity range of the latter is $-25 \frac{\mathrm{m}}{\mathrm{s}}<U_{\mathrm{Lo}}<5 \frac{\mathrm{m}}{\mathrm{s}}$ and it contains smaller droplets, while the velocity range of the Hi-population with slightly bigger droplets is depending on the spray position. In Fig. 15, all the measurement positions, where both droplet populations are separable, are colored blue. They are congruent with the recirculation zones visible in Figs. 10 and 12 as dark structures.

Based on these observations, we propose the hypothesis that the droplets of the Lo-population follow the spray's gas flow which is characterized by recirculation zones due to shock systems and the resulting upstream droplet motion. The droplets of the Hi-population follow instead mainly the original flow trajectories determined by the flow conditions in the injector. The spatial distribution of the number of valid Doppler-bursts separated for each population is depicted in Fig. 16 and supports this hypothesis, too: Especially at the close-injector positions $5<y / D<30$, the number of valid droplets differ by up to two orders of magnitude between the two populations, with the lower number within

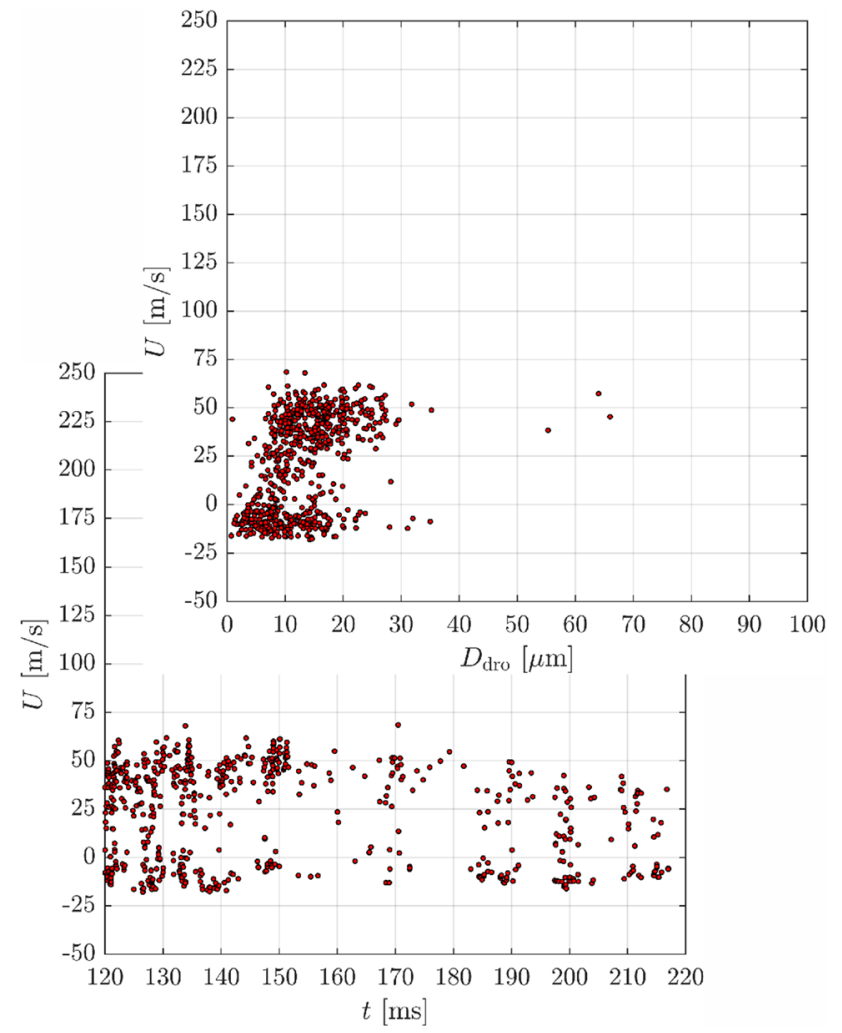

Fig. 14 Vertical velocity $U$ as function of arrival time $t$ and of measured droplet diameter $D_{\text {dro }}$ at position $x / D=0$ and $y / D=30$ in a fully flashing LN2 spray (Rees et al. 2019a)

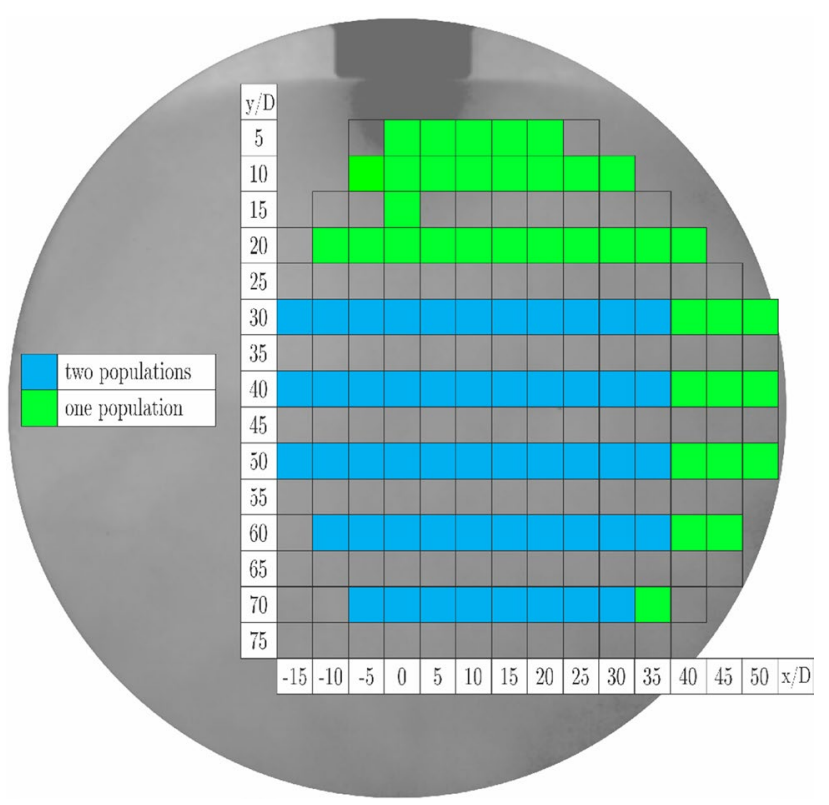

Fig. 15 Positions in fully flashing LN2 sprays with Hi- and Lo-populations; injection conditions see Table 2 

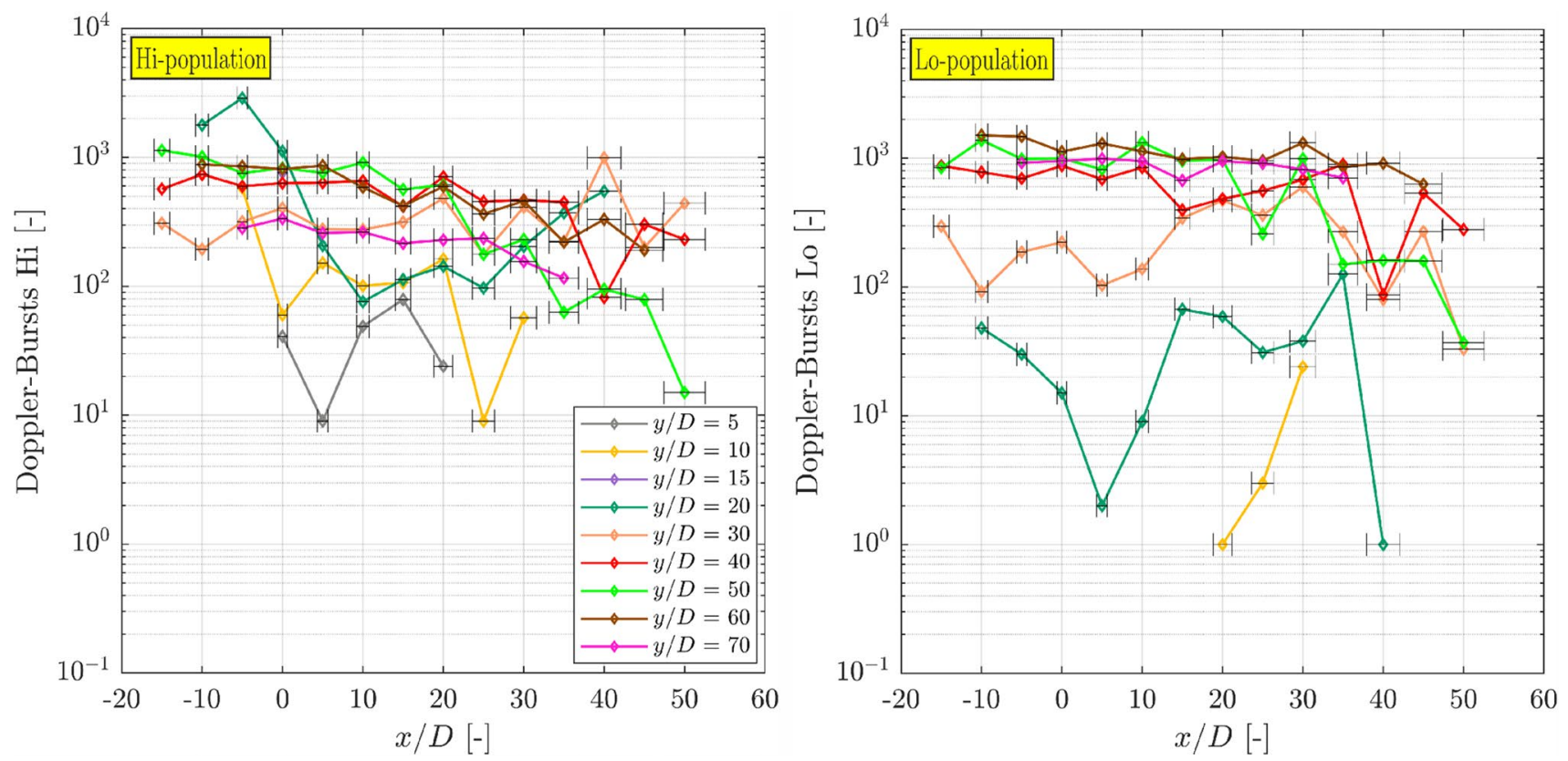

Fig. 16 Spatial distributions of the number of valid Doppler-bursts separated for the droplet populations Hi (left) and Lo (right) in fully flashing LN2 sprays; injection conditions see Table 2

the Lo-population. At the closest measurement position to the injector $y / D=5$, no droplets within that population were measured as can be seen on the right-hand side of Fig. 16. This means, that close to the injector the majority of the measured droplets are fast and big ones. Only when the distance to the injector is growing and the recirculation zones are reached at about $y / D>30$, the number of smaller droplets with the low and negative vertical velocities is rising until their numbers are similar to the ones of the Hi-population for $30<y / D<60$. In contrast, at the position $y / D=70$, there are less droplets within the Hi-population because most of the droplets with initial injector conditions have been partly evaporated and influenced by droplet interactions in the recirculation zones.

\subsubsection{Mean vertical velocity distributions}

Since the spatial distribution of the vertical velocity component $U$ in Sect. 4.1 (s. Fig. 7) was determined by averaging the entire droplet population, we separately averaged the Hi- and Lo-population to get the respective distribution for each, see Fig. 17 with the vertical velocity distribution of the Hi-population on the left-hand side and the one of the Lo-population on the right-hand side. The evolution of the vertical velocity profiles $U_{\mathrm{Hi}}$ for the Hi-population differ only slightly from the entire population on the left-hand side of Fig. 7 at positions close to the injector due to the low number of droplets in the Lo-population in this region. Further downstream at $y / D \approx 30$ the sudden velocity drop is much smaller now. This is because the droplets of the Lo-population reduced the mean values of the entire population in contrast to the averaging of the Hi-population without those reducing droplets. Hence, the mean vertical velocities of the Hi-population at $y / D=70$ are about $U_{\mathrm{Hi}} \approx 17.4 \frac{\mathrm{m}}{\mathrm{s}}$, which is close to the initial flow velocity of $u_{\mathrm{inj}}=18.1 \frac{\mathrm{m}}{\mathrm{s}}$ in the injector. These trends can be seen at the axial velocity profile $U_{\text {axis }}$ in Fig. 8 , too. Here, the profile of the Hi-population is much closer to the ethanol data of Kamoun et al. (2012) and Kamoun (2015) compared to the entire droplet population. The deceleration of the big droplets within the Hi-population is dampened due to the on-going evaporation at the droplet surface and the resulting increase in their kinetic energy.

The vertical velocity profiles of the Lo-population $U_{\mathrm{Lo}}$ are very homogeneous compared to the Hi-population, especially at the positions $30 \leq y / D \leq 70$. In particular close to the spray axis, the negative velocities are almost constant with $U_{\mathrm{Lo}} \approx-7 \frac{\mathrm{m}}{\mathrm{s}}$. Only for radial distances of about $x / D \geq 20$, a slight trend towards positive, but still low velocities is visible.

\subsubsection{Mean horizontal velocity distributions}

Because of the already low horizontal velocities in the regions of the recirculation zones compared to the vertical ones, no separate droplet populations of different velocity or diameter classes were found. 

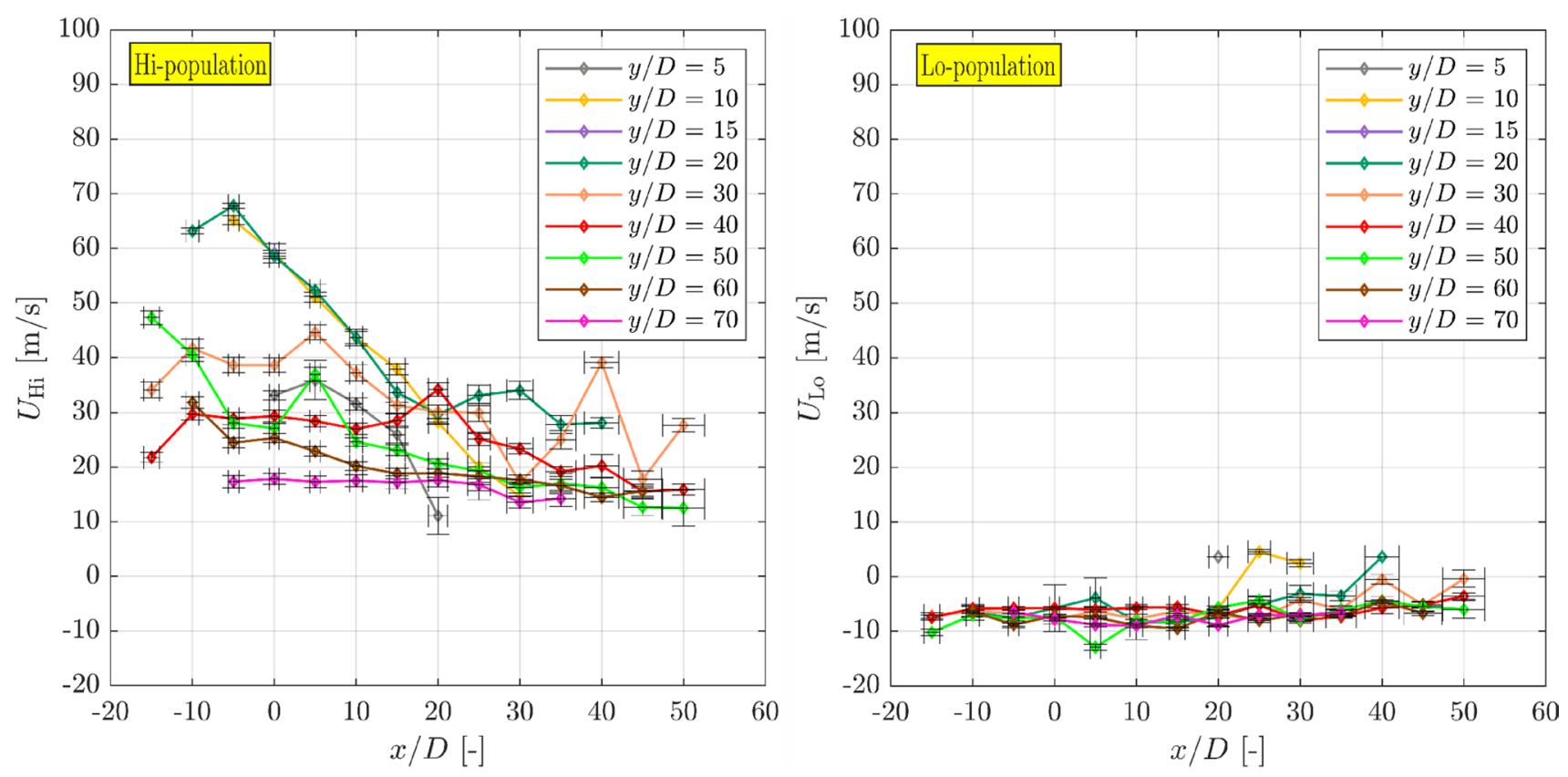

Fig. 17 Spatial distributions of the vertical velocity component $U$ separated for the droplet populations Hi (left) and Lo (right) in fully flashing LN2 sprays; injection conditions see Table 2

\subsubsection{Mean diameter distributions}

The separate evaluation of the two populations in terms of the AMDs results in the spatial distributions on the lefthand side in Fig. 18 for the Hi-population and on the righthand side for the Lo one. Obviously, in the Hi-population, the majority of droplets has AMDs with $D_{10, \mathrm{Hi}}>10 \mu \mathrm{m}$. Only close to the injector with $y / D<20$ as well as at the outer regions of the profile $y / D=30$ smaller mean values were determined. In contrast, mainly droplets with AMDs of $D_{10, \text { Lo }} \approx 10 \mu \mathrm{m}$ or less were found in the Lo-population. Close to the injector and in the outer regions the droplets exceed this value up to mean diameters of about $D_{10, \mathrm{Lo}} \approx 19 \mu \mathrm{m}$. This means that close to the injector and towards the periphery of the spray either small and fast droplets exist or bigger and slower ones.

The influence of recirculation zones at $y / D \approx 70$ can be seen at profile $y / D=70$ of the Hi-population with AMDs of $13.7 \mu \mathrm{m} \leq D_{10, \mathrm{Hi}} \leq 16.1 \mu \mathrm{m}$. According to the left-hand side of Fig. 16, the number of Hi-population droplets in this region with values of about $100-300$ is, first, lower than at the profiles directly above, like e.g. at $y / D=60$. Second, it is also lower than the droplet number at the same profile in the Lo-population with values of roughly 700-1000, which here are similar to its profiles above. But with AMDs of $8.6 \mu \mathrm{m} \leq D_{10, \mathrm{Lo}} \leq 10.4 \mu \mathrm{m}$ the droplets on average are smaller. Hence, it can be concluded that a few big secondary droplets originating from recirculations at the spray margins adopt the kinetics of the initial flow conditions of the injector flow due to droplet interactions. On the other hand, the high number of small Lo-population droplets at $y / D=70$ follows the gas flow characterized by flash boiling flow conditions.

Besides the axial diameter profile at $x / D=0$ of the entire population, the axial AMD profiles $D_{10 \text {,axis,Hi }}$ and $D_{10 \text {,axis,Lo }}$ as well as the SMD profiles $D_{32, \text { axis,Hi }}$ and $D_{32 \text {,axis,Lo }}$ are depicted on the left-hand and right-hand side, respectively, in Fig. 13 for comparison with the R-134A study by Yildiz et al. (2004). The absence of small and slow particles belonging to the Lo-population close to the injector orifice is in this chart even more obvious than in the entire spatial distributions. Those kind of droplets with AMDs of $D_{10 \text {,axis,Lo }}=11.6 \mu \mathrm{m}$ were measured only for axial distances of $y / D \geq 20$. With growing distance their AMDs decrease, only slightly to a minimum of about $D_{10 \text {,axis,Lo }}=8.0 \mu \mathrm{m}$. The axial AMD profile of the Hi-population lies above the one of the entire droplet population and is much closer to the one of Yildiz et al., especially for $20 \leq y / D \leq 50$. By means of the SMD evolution on the right-hand side of Fig. 13, the influence of the recirculation zones for $y / D>50$ can be visualized. Here, the SMD of the Lo-population with $D_{32 \text {,axis,Lo }} \approx 26 \mu \mathrm{m}$ is higher than the one of the Hi-population with $D_{32 \text {,axis,Hi }} \approx 22 \mu \mathrm{m}$ and is further away from the respective AMD evolution. This means that the influence of the recirculation zones is higher for the Lopopulation, resulting in a more inhomogeneous and less monodisperse spray pattern in those regions. 

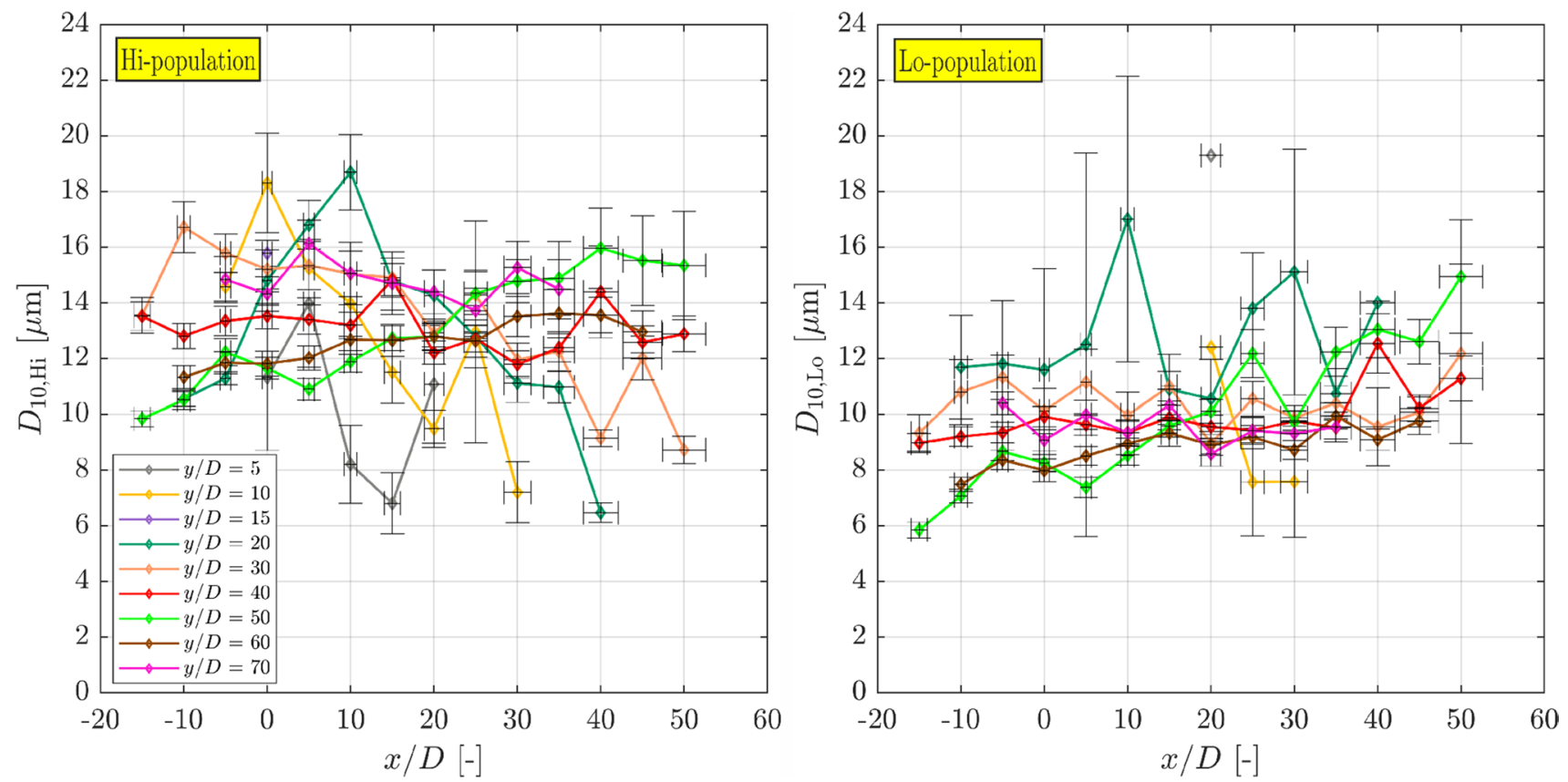

Fig. 18 Spatial distributions of the AMD $=D_{10}$ separated for the droplet populations Hi (left) and Lo (right) in fully flashing LN2 sprays; injection conditions see Table 2

\section{Summary and conclusion}

At the new cryogenic test bench, M3.3 with a temperature controlled injection system we analyzed highly superheated liquid nitrogen sprays by means of laser-based 2D Phase Doppler diagnostics and by high-speed shadowgraphy. To determine the spatial distributions of the vertical velocities, the horizontal velocities and the diameters of the spray droplets, we kept constant the injection conditions in terms of temperature, pressure and injector geometry, while the measurement positions of the Phase Doppler optics were varied by a traverse system. The local vertical and horizontal velocity distributions show their maximum values close to the injector exit. Hence, this core region of the liquid nitrogen spray is a zone of high kinetic energy, where the internal energy is transformed into kinetic one with the resulting spray expansion due to evaporation. The diameter measurements in this high energy zone revealed the difficulty of the optical accessibility to these dense core regions as observed multiple times for storable fluids in the literature. Furthermore, the small of-axis angle of $12^{\circ}$ of the used PDA set-up leads to a biased diameter determination especially for small droplets and results in an averaged systematical error of $26 \%$. Hence, the optical set-up and accessibility to the sprays has to be modified for future measurement campaigns in order to allow higher off-axis angles for a more reliable size determination. The likely existence of non-spherical liquid structures and ligaments and especially the high optical densities in the core regions make it even more difficult to get reliable droplet size data in that kind of spray regions. However, with increasing axial distance to the injector orifice, the sprays develop a more and more monodisperse pattern with almost homogeneous droplet mean diameter distributions. We observed two droplet populations with droplets varying in their direction of motion, velocity magnitude and diameters in most parts of the flash boiling sprays. This shows the existence of several recirculation zones in the spray. They can occur due to shock systems as a consequence of flash boiling kinetics and due to external influences out of the optical access field of view like e.g. the interaction of chilled down droplets from the outer spray regions with the walls of the vacuum chamber.

The experimental data of flash boiling liquid nitrogen generated within this study provide a comprehensive data base for the validation of numerical models and further numerical investigations. Furthermore, the test campaigns with liquid nitrogen prepare the investigation of flash boiling of actual rocket propellants like liquid oxygen or liquid methane later on at this test bench.

Acknowledgements Open Access funding provided by Projekt DEAL. We kindly acknowledge the financial support by the Deutsche Forschungsgemeinschaft (DFG, German Research Foundation) within the project SFB-TRR 75, project number 84292822. 


\section{Compliance with ethical standards}

Conflict of interest The authors declare that they have no conflict of interest.

Open Access This article is licensed under a Creative Commons Attribution 4.0 International License, which permits use, sharing, adaptation, distribution and reproduction in any medium or format, as long as you give appropriate credit to the original author(s) and the source, provide a link to the Creative Commons licence, and indicate if changes were made. The images or other third party material in this article are included in the article's Creative Commons licence, unless indicated otherwise in a credit line to the material. If material is not included in the article's Creative Commons licence and your intended use is not permitted by statutory regulation or exceeds the permitted use, you will need to obtain permission directly from the copyright holder. To view a copy of this licence, visit http://creativecommons.org/licenses/by/4.0/.

\section{References}

Abramovich GN (1963) The theory of turbulent jets, 1st edn. The MIT Press, Cambridge

Aleiferis PG, Serras-Pereira J, Augoye A, Davies TJ, Cracknell RF, Richardson D (2010) Effect of fuel temperature on in-nozzle cavitation and spray formation of liquid hydrocarbons and alcohols from a real-size optical injector for direct-injection spark-ignition engines. Int J Heat Mass Transf 53(21-22):4588-4606

Araneo L, Dondè R (2017) Flash boiling in a multihole G-DI injector-effects of the fuel distillation curve. Fuel 191:500-510

Araneo L, Dondè R (2019) Atomization of a G-DI spray with air dissolved in gasoline and mono-component fuels. In: 29th ILASSEurope conference, Paris, France

Ashgriz N, Poo JY (1990) Coalescence and separation in binary collisions of liquid drops. J Fluid Mech 221:183-204

Brown R, York JL (1962) Sprays formed by flashing liquid jets. AIChE J 8(2):149-153

Cleary V, Bowen PJ, Witlox HWM (2007) Flashing liquid jets and twophase droplet dispersion: I. Experiments for derivation of droplet atomisation correlations. J Hazard Mater 142(3):786-796

De Rosa M, Sender J, Zimmermann H, Oschwald M (2006) Cryogenic spray ignition at high altitude conditions. In: 42nd AIAA/ASME/ SAE/ASEE joint propulsion conference, Sacramento, California

Elias E, Chambré PL (1993) Flashing inception in water during rapid decompression. J Heat Transf 115(1):231-238

Franquet E, Perrier V, Gibout S, Bruel P (2015) Free underexpanded jets in a quiescent medium: a review. Prog Aerosp Sci 77:25-53

Gärtner JW, Kronenburg A, Rees A, Sender J, Oschwald M, Lamanna G (2020) Numerical and experimental analysis of flashing cryogenic nitrogen. Int J Multiph Flow 130:103360

Gemci T, Yakut K, Chigier N, Ho TC (2004) Experimental study of flash atomization of binary hydrocarbon liquids. Int J Multiph Flow 30(4):395-417

Johns HE, Wilhelm JO (1937) The refractive indices of liquid oxygen, nitrogen, and hydrogen. Can J Res 15(7):101-108

Kamoun H (2015) Flash-atomization and vaporization at near vacuum conditions. Dissertation, Universität Stuttgart

Kamoun H, Ruberto S, Lamanna G, Weigand B, Steelant J (2012) Velocity characterisation of a flashing ethanol jet with laser correlation velocimetry. In: 24th ILASS-Americas conference, San Antonio, Texas

Kurschat TH, Chaves H, Meier GEA (1992) Complete adiabatic evaporation of highly superheated liquid jets. J Fluid Mech 236:43-59
Lamanna G, Kamoun H, Weigand B, Steelant J (2014) Towards a unified treatment of fully flashing sprays. Int J Multiph Flow $58: 168-184$

Lamanna G, Kamoun H, Weigand B, Manfletti C, Rees A, Sender J, Oschwald M, Steelant J (2015) Flashing behavior of rocket engine propellants. At Spray 25(10):837-856

Lecourt R, Barricau P (2009) Spray velocity and drop size measurements in flashing conditions. At Spray 19(2):103-133

Luo M, Haidn OJ (2016) Characterization of flashing phenomena with cryogenic fluid under vacuum conditions. J Propuls Power $3(5): 1253-1263$

Manfletti C (2014) Laser ignition of an experimental 400N cryogenic reaction and control thruster: pre-ignition conditions. J Propuls Power 30(4):925-933

Medrano FF, Fukumoto Y, Velte CM, Hodžić A (2017) Mass entrainment rate of an ideal momentum turbulent round jet. J Phys Soc Jpn 86(034401):1-10

Meyer J (1911) Zur Kenntnis des negativen Drucks in Flüssigkeiten. Abh Dtsch Bunsen-Ges Phys Chem 3(1):1-53

Simões Moreira JR, Vieira MM, Angelo E (2002) Highly expanded flashing liquid jets. J Thermophys Heat Transf 16(3):415-424

Rees A, Araneo L, Salzmann H, Kurudzija E, Suslov D, Lamanna G, Sender J, Oschwald M (2019a) Investigation of velocity and droplet size distributions of flash boiling LN2-jets with phase doppler anemometry. In: 29th ILASS-Europe conference, Paris, France

Rees A, Salzmann H, Sender J, Oschwald M (2019b) Investigation of flashing LN2-jets in terms of spray morphology, droplet size and velocity distributions. In: 8th EUCASS conference, Madrid, Spain

Reitz RD (1990) A photographic study of flash-boiling atomization. Aerosol Sci Technol 12(3):561-569

Senda J, Hojyo Y, Fujimoto H (1994) Modeling on atomization and vaporization process in flash boiling spray. JSAE Rev 15(4):291-296

Senda J, Wada Y, Kawano D, Fujimoto H (2008) Improvement of combustion and emissions in diesel engines by means of enhanced mixture formation based on flash boiling of mixed fuel. Int $\mathbf{J}$ Engine Res 9(1):15-27

Simões-Moreira JR, Vieira MM, Angelo E (2002) Highly expanded flashing liquid jets. J Thermophys Heat Transf 16(3):415-424

Tropea C, Xu TH, Onofri F, Gréhan G, Haugen P, Stieglmeier M (1996) Dual-mode phase-Doppler anemometer. Part Part Syst Charact 13:165-170

Wismer KL (1921) The pressure-volume relation of super-heated liquids. J Phys Chem 26(4):301-315

Witlox HWM, Bowen PJ (2002) Flashing liquid jets and two-phase dispersion: a review. Contract Research Report 403/2002, HSE

Witlox HWM, Harper M, Bowen PJ, Cleary V (2007) Flashing liquid jets and two-phase droplet dispersion: II. Comparison and validation of droplet size and rainout formulations. J Hazard Mater 142(3):797-809

Yildiz D, Rambaud P, Van Beeck J, Buchlin JM (2002) A study on the dynamics of a flashing jet. Final Contract Research Report EAR0030, VKI

Yildiz D, Van Beeck J, Riethmuller ML (2004) Feasibility exploration of laser-based techniques for characterization of a flashing jet. Part Part Syst Charact 21(5):390-402

Zeng Y, Lee CFF (2001) An atomization model for flash boiling sprays. Combust Sci Technol 169(1):45-67

Publisher's Note Springer Nature remains neutral with regard to jurisdictional claims in published maps and institutional affiliations. 


\section{Affiliations}

\section{Andreas Rees $^{1}$ (1) $\cdot$ Lucio Araneo $^{2} \cdot$ Heiko Salzmann $^{1} \cdot$ Grazia Lamanna $^{3} \cdot$ Joachim Sender $^{1} \cdot$ Michael Oschwald $^{1,4}$}

1 Institute of Space Propulsion, DLR, Langer Grund, 74239 Hardthausen a.K., Germany

2 Energy Department, Politecnico di Milano, Via Lambruschini 4A, 20156 Milan, Italy

3 Institute of Aerospace Thermodynamics, University of Stuttgart, Pfaffenwaldring 31, 70569 Stuttgart, Germany
4 Institute of Jet Propulsion and Turbomachinery, RWTH Aachen University, Templergraben 55, 52062 Aachen, Germany 